\title{
A Quasi-Optimal Non-Overlapping Domain Decomposition Algorithm for the Helmholtz Equation
}

\author{
Y. Boubendir* \\ X. Antoine \\ C. Geuzaine ‡ $^{\ddagger}$
}

February 23, 2011

\begin{abstract}
This paper presents a new non-overlapping domain decomposition method for the Helmholtz equation, whose effective convergence is quasi-optimal. These improved properties result from a combination of an appropriate choice of transmission conditions and a suitable approximation of the Dirichlet to Neumann operator. A convergence theorem of the algorithm is established and numerical results validating the new approach are presented in both two and three dimensions.
\end{abstract}

\section{Introduction}

In this paper, we are interested in non-overlapping Domain Decomposition Methods (DDMs) for the Helmholtz equation. Such methods were introduced by Lions [36] for the Laplace equation and extended to the Helmholtz equation by Després [19, 20, 21]. Essentially, the method consists in combining the continuity conditions (of the field and its normal derivative) on the artificial interfaces between subdomains, in order to obtain Robin boundary conditions and to solve the overall problem by iterating over the subdomains [39, 47, 45]. Robin conditions (also called absorbing or impedance boundary conditions) are chosen to couple the subdomains because using the natural conditions leads to divergent iterative algorithms [12].

Improving the convergence properties of the iterative process constitutes the key in designing effective algorithms, in particular in mid and high frequency. The optimal convergence is obtained by defining the transmission conditions, on each interface, using the Dirichlet-to-Neumann (DtN) operator corresponding to the problem sharing the same interface $[41,40]$. This however leads to a very expensive procedure in practice. The first contribution following the original method of Després, using particular non-local transmission conditions, was made in [17] with a relative success regarding the effective convergence. A great variety of techniques based on local transmission conditions have also been proposed to improve the convergence: these include the class of FETI-H methods [24, 25, 26, 14], the optimized Schwartz approach [27], and the evanescent modes damping algorithm [15, 16, 12]. However, the related impedance operators do not accurately approximate the exact DtN operator on all the modes of the solution, which makes the resulting iterative methods suboptimal.

In this paper, we propose a new square-root based transmission condition, localized using Padé approximants, which accurately approximates the DtN operator and allows to design an algorithm with

\footnotetext{
* Department of Mathematical Sciences and Center for Applied Mathematics and Statistics, NJIT, Univ. Heights. 323 Dr. M. L. King Jr. Blvd, Newark, NJ 07102, USA.

${ }^{\dagger}$ Institut Elie Cartan Nancy (IECN), Nancy University, INRIA Corida Team, B.P. 239, F-54506 Vandoeuvre-lès-Nancy Cedex, France.

${ }^{\ddagger}$ University of Liège, Department of Electrical Engineering and Computer Science, Montefiore Institute B28, B-4000 Liège, Belgium.
} 


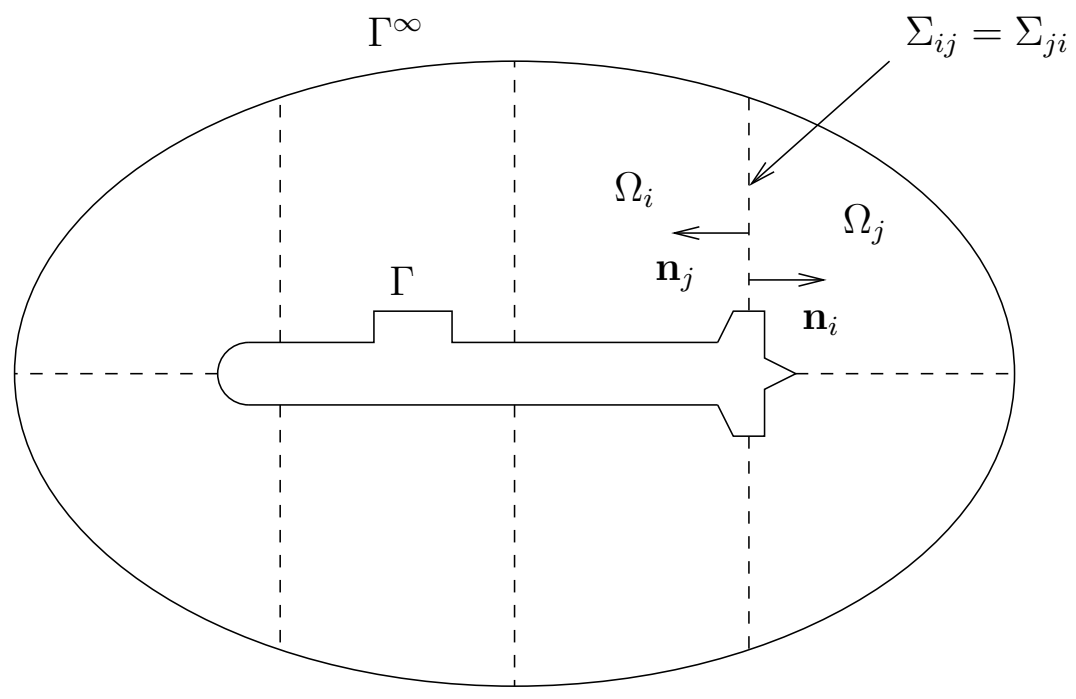

Figure 1: Example of a two-dimensional non-overlapping domain decomposition method.

quasi-optimal convergence properties. Indeed, we will show that the rate of convergence is optimal on the evanescent modes and is significantly improved compared to current techniques for the remaining modes. In the effective convergence, this results in a DDM independent of the wavenumber as well as the mesh discretization. Moreover, we will see how the resulting approximate DtN operator is easy to implement in a basic finite element solver.

The paper is organized as follows. In Section 2, we introduce the scattering problem as well as the nonoverlapping DDM. We present in the third Section a non-local square-root operator which approximates the exact DtN transmission operator. Section 4 develops a convergence analysis for this approximate transmission condition on a model problem. Section 5 details the complex Padé approximation of the square-root operator to get a local representation. Section 6 presents the finite element implementation of the resulting DDM. Numerical results on both two- and three-dimensional problems are presented in Section 7.

\section{Scattering Problem and Non-Overlapping Domain Decompo- sition Method}

Let us consider the three-dimensional time-harmonic scattering problem of an incident acoustic wave by an obstacle $K$. We want to compute the scattered field $u$ solution to the exterior Helmholtz equation with a Dirichlet boundary condition ${ }^{1}$ :

$$
\left\{\begin{array}{l}
\Delta u+k^{2} u=0 \quad \text { in } \mathbb{R}^{3} \backslash K, \\
u=f \text { on } \Gamma=\partial K \\
\lim _{|\mathbf{x}| \rightarrow \infty}|\mathbf{x}|\left(\partial_{|\mathbf{x}|} u-\imath k u\right)=0 .
\end{array}\right.
$$

The boundary data $f$ is fixed by a plane wave: $f=-e^{\imath k \boldsymbol{\alpha} \cdot \mathbf{x}}$, with $\mathbf{x}=\left(x_{1}, x_{2}, x_{3}\right) \in \mathbb{R}^{3}$ and $\imath=\sqrt{-1}$. The incidence angle $\boldsymbol{\alpha}$ is normalized on the unit sphere $(|\boldsymbol{\alpha}|=1)$ and $k$ denotes the wavenumber, related

\footnotetext{
${ }^{1}$ The Dirichlet boundary condition models a sound-soft obstacle; Neumann (sound-hard) or Fourier-Robin (impedance) boundary conditions may also be set, which would not fundamentally change the rest of the paper.
} 
to the wavelength $\lambda$ of the incident wave through $k=2 \pi / \lambda$. The last equation of system (1) is the Sommerfeld radiation condition at infinity, which imposes that the scattered wave is outgoing.

To solve (1), we combine Absorbing Boundary Conditions (ABCs) with Lions-Després' non-overlapping domain decomposition method. The ABC method consists in truncating the infinite domain by introducing a fictitious boundary $\Gamma^{\infty}$ to get a bounded computational region. Then, system (1) is approximated by

$$
\left\{\begin{array}{l}
\Delta u+k^{2} u=0 \text { in } \Omega, \\
u=f \text { on } \Gamma, \\
\partial_{\mathbf{n}} u+\mathcal{B} u=0 \text { on } \Gamma^{\infty},
\end{array}\right.
$$

where $\Omega$ is the bounded domain enclosed by the fictitious boundary $\Gamma^{\infty}$ and $\Gamma$ (see Figure 1) and where the operator $\mathcal{B}$ represents an approximation of the DtN operator (for example $\mathcal{B}=-\imath k$ ) on $\Gamma^{\infty}$. The vector $\mathbf{n}$ is the outwardly directed unit normal to $\Gamma^{\infty}$. Let us remark that all what follows can be adapted to the case of other truncation techniques, like e.g. perfectly matched layers (PMLs) [13, 18, 44, 48].

The first step of the Lions-Després domain decomposition method [36, 19] consists in splitting $\Omega$ into several subdomains $\Omega_{i}, i=1, \ldots, N_{\text {dom }}$, such that (see Figure 1 ):

- $\bar{\Omega}=\bigcup_{i=1}^{N_{\text {dom }}} \bar{\Omega}_{i}\left(i=1, \ldots, N_{\text {dom }}\right)$,

- $\Omega_{i} \cap \Omega_{j}=\emptyset$, if $i \neq j,\left(i, j=1, \ldots, N_{\text {dom }}\right)$,

- $\partial \Omega_{i} \cap \partial \Omega_{j}=\bar{\Sigma}_{i j}=\bar{\Sigma}_{j i}\left(i, j=1, \ldots, N_{\text {dom }}\right)$ is the artificial interface separating $\Omega_{i}$ and $\Omega_{j}$ as long as its interior $\Sigma_{i j}$ is not empty.

The second step consists in reducing the solution of the initial problem (2) by solving the local transmission problems

$$
\begin{aligned}
& \left\{\begin{array}{l}
\Delta u_{i}^{(n+1)}+k^{2} u_{i}^{(n+1)}=0 \text { in } \Omega_{i}, \\
u_{i}^{(n+1)}=f_{i} \text { on } \Gamma_{i}, \\
\partial_{\mathbf{n}_{i}} u_{i}^{(n+1)}+\mathcal{B} u_{i}^{(n+1)}=0 \text { on } \Gamma_{i}^{\infty},
\end{array}\right. \\
& \partial_{\mathbf{n}_{i}} u_{i}^{(n+1)}+\mathcal{S} u_{i}^{(n+1)}=g_{i j}^{(n)} \text { on } \Sigma_{i j},
\end{aligned}
$$

and then in forming the quantities to be transmitted through the interfaces

$$
g_{i j}^{(n+1)}=-\partial_{\mathbf{n}_{j}} u_{j}^{(n+1)}+\mathcal{S} u_{j}^{(n+1)}=-g_{i j}^{(n)}+2 \mathcal{S} u_{j}^{(n+1)} \quad \text { on } \Sigma_{i j},
$$

where $u_{i}=\left.u\right|_{\Omega_{i}}, \mathbf{n}_{i}$ (resp. $\mathbf{n}_{j}$ ) is the outward unit normal to the boundary of $\Omega_{i}$ (resp. $\Omega_{j}$ ), $i=$ $1, \ldots, N_{\text {dom }}, j=1, \ldots, N_{\text {dom }}, \Gamma_{i}=\partial \Omega_{i} \cap \Gamma, \Gamma_{i}^{\infty}=\partial \Omega_{i} \cap \Gamma^{\infty}$ and $\mathcal{S}$ an invertible operator. Let us note that the boundary condition on $\Gamma_{i}$ (resp. $\Gamma_{i}^{\infty}$ ) does not take place if the interior of $\partial \Omega_{i} \cap \Gamma$ (resp. $\left.\partial \Omega_{i} \cap \Gamma^{\infty}\right)$ is the empty set. We will assume in all that follows that the DDM is well-posed, in the sense that each subproblem (3a)-(3b) is itself well-posed, i.e., away from interior resonances.

Solving at each step all the local transmission problems through (3)-(4) may be recast as one application of the iteration operator $\mathcal{A}: \times_{i, j=1}^{N_{\text {dom }}} L^{2}\left(\Sigma_{i j}\right) \rightarrow \times_{i, j=1}^{N_{\text {dom }}} L^{2}\left(\Sigma_{i j}\right)$ [11] defined by

$$
g^{(n+1)}=\mathcal{A} g^{(n)}+b,
$$

where $g^{(n)}$ is the set of boundary data $\left(g_{i j}^{(n)}\right)_{1 \leq i, j \leq N_{\mathrm{dom}}}$, and $b$ is given by the Dirichlet boundary condition. Therefore, (3)-(4) can be seen as an iteration of the Jacobi method (or fixed point iteration) applied to the linear system:

$$
(I-\mathcal{A}) g=b,
$$

where $I$ is the identity operator. Following this idea, any Krylov solver could also be applied to solve this equation. Indeed, we will see that the GMRES algorithm significantly improves the iterative process over the successive approximations procedure (5). 


\section{Square-Root Based Transmission Condition}

It is well-known that the convergence of the domain decomposition method for scattering problems strongly depends on the choice of the transmission operator $\mathcal{S}$. Indeed, to each choice of operator $\mathcal{S}$ corresponds an iteration operator $\mathcal{A}$ with particular spectral properties. Several techniques were developed to improve the convergence by modifying the original algorithm introduced by Després in [19]. In this last reference, the low-order approximation of the DtN operator

$$
\mathcal{S}^{0} u=-\imath k u
$$

is used. For this choice of operator $\mathcal{S}$, it can be shown [15] that the resulting iteration operator only acts on the part of the spectrum corresponding to the propagating modes while the eigenvalues related to the evanescent modes have unit modulus [15]. This directly impacts the convergence (divergence) properties of the resulting iterative scheme. Two families of techniques have been proposed to overcome this problem. First, algorithms based on the optimization of the rate of convergence were introduced by Gander et al. [27], where improved local second-order approximations of the DtN map with optimized coefficients are used. In this case, the local transmission operators take the following general form for a generic transmitting boundary $\Sigma$ :

$$
\mathcal{S}^{\mathrm{oo} 2} u=-\alpha \operatorname{div}_{\Sigma}\left(A \nabla_{\Sigma} u\right)+a u,
$$

where $A$ and $a$ are a surface tensor (which can be zero, leading to the usual Després approach) and a surface function, respectively, that can be optimized according to the rate of convergence. The operator $\operatorname{div}_{\Sigma}$ in (8) is the surface divergence of a tangent vector field on $\Sigma$ and $\nabla_{\Sigma}$ is the tangential gradient of a surface field. Second, the Evanescent Modes Damping Algorithm (EMDA) was introduced by Boubendir in $[15,16]$, with the explicit aim to damp the evanescent modes:

$$
\mathcal{S}^{\mathcal{X}} u=-\imath k u+\mathcal{X} u
$$

where $\mathcal{X}$ is a self-adjoint positive operator. We only consider here the usual case where $\mathcal{X}$ is a real-valued positive coefficient.

In this paper we propose a new "square-root" transmission operator [6] that takes the following form:

$$
S^{\mathrm{sq}, \varepsilon} u=-\imath k \sqrt{1+\operatorname{div}_{\Sigma}\left(\frac{1}{k_{\varepsilon}^{2}} \nabla_{\Sigma}\right)} u
$$

where

$$
k_{\varepsilon}=k+\imath \varepsilon
$$

is a complexified wavenumber, and where the square-root $\sqrt{A}$ of an operator $A$ is classically defined through the spectral decomposition of $A$ [46]. Furthermore, the notation $\sqrt{z}$ designates the principal determination of the square-root of a complex number $z$ with branch-cut along the negative real axis. We will show in what follows that

1. the nonlocal operator $S^{\mathrm{sq}, \varepsilon}$ can be accurately localized using complex Padé approximants, and suitably combined with finite element methods;

2. the convergence of the resulting DDM is quasi-optimal: the rate of convergence corresponding to the evanescent modes is zero, and significantly improved for the remaining modes. This results in an effective solution where the iterative procedure is quasi independent of both the wavenumber and the level of accuracy of the spatial discretization (mesh refinement). 
The choice of this square-root operator is motivated by developments of Absorbing Boundary Conditions $(\mathrm{ABC})$ for scattering problems, where the goal is to minimize reflection at the fictitious surface $\Gamma^{\infty}$. In the eighties, the Engquist-Majda [23] and Bayliss-Turkel $[9,2,10]$ ABCs were developed, which are written as

$$
\partial_{\mathbf{n}} u+M u=0 \quad \text { on } \Gamma^{\infty},
$$

where $M$ is a local approximation of the DtN operator that takes a form similar to (8). While these conditions are widely used for practical computations and most particularly for engineering purposes, it was recently shown in [6] that they also do not model the evanescent and grazing modes, resulting in a loss of accuracy of the method. In order to solve this problem, a high-order local ABC was introduced in [6,34], which uses $M=S^{\mathrm{sq}, \varepsilon}$ in (12) to model all the scattering modes: propagating, evanescent as well as (in an approximate way) grazing. This $\mathrm{ABC}$ can be localized with complex Padé approximants, and the coefficient $\varepsilon$ in (11) can then be chosen to minimize spurious reflections at the boundary.

Without giving too much details about the derivation of the operator $S^{\mathrm{sq}, \varepsilon}$ in (10), let us briefly explain where it comes from (see [6] for more details). To obtain (10), we compute for a general surface $\Sigma$ the principal symbol $\sigma_{1}\left(=-\imath k \sqrt{1-|\boldsymbol{\xi}|^{2} / k^{2}}\right)$ [46] of the exact DtN operator $\Lambda$ [2]. (The covariable $\boldsymbol{\xi}=\left(\xi_{1}, \xi_{2}\right)$ is roughly the Fourier variable locally to a point of the surface after a local mapping [2], with $|\boldsymbol{\xi}|^{2}=\xi_{1}^{2}+\xi_{2}^{2}$.) If we denote by $\sigma$ the total symbol [46] of $\Lambda$, it can be proved by using a factorization theorem [2] at the microlocal level that we have an asymptotic expansion: $\sigma \approx \sigma_{1}+\sigma_{R}$, where $\sigma_{R}$ is a remaining symbol of order less than one and therefore has an associated pseudodifferential operator of the same order. Then, we only retain the principal symbol $\sigma_{1}$ and do the approximation: $\Lambda \approx \mathrm{Op}\left(\sigma_{1}\right)$, where $\mathrm{Op}\left(\sigma_{1}\right)$ designates the pseudodifferential operator with symbol $\sigma_{1}$. In practice, for a general surface, it is difficult to write more precisely the corresponding operator. However, it can be proved [7] that, up to lower order symbols, the principal symbol of the square-root operator $-\imath k \sqrt{1+\operatorname{div}_{\Sigma}\left(\frac{1}{k_{\varepsilon}^{2}} \nabla_{\Sigma}\right)}$ is equal to $\sigma_{1}$, for $\varepsilon=0$. Therefore, since we do the approximation modulo a lower order operator, we can approximate $\Lambda$ by $-\imath k \sqrt{1+\operatorname{div}_{\Sigma}\left(\frac{1}{k_{\varepsilon}^{2}} \nabla_{\Sigma}\right)}$ and get the corresponding square-root transmission boundary condition. The damping parameter $\varepsilon$ is then introduced to regularize the square-root operator with the aim to model more correctly the frequencies within the transition zone (called glancing zone in microlocal analysis [2]). Let us remark that the derivation is similar to Beam Propagation Methods (BPMs) for bent optical waveguides $[7,37,30]$, and that the theoretical factorization theorem used in the preceeding derivation allows to split the outgoing and incoming parts of the wave field through transmission boundary conditions $[2,7]$.

The Després and EMDA transmission operators (7) and (9) are particular cases of Impedance Boundary Conditions (IBC) [43]. The second order (8) and the new square-root operator (10) (as well as its Padé localization) fall into the realm of so-called Generalized Impedance Boundary Conditions (GIBC) [43]. In what follows we will thus refer to the transmission conditions related to the Després and EMDA algorithms by $\operatorname{IBC}(0)$ and $\operatorname{IBC}(\mathcal{X})$, respectively, and to the square-root transmission condition by $\operatorname{GIBC}(\mathrm{sq}, \varepsilon)$.

\section{Convergence Analysis for a Model Problem}

In order to study the convergence of the proposed DDM with square-root transmission operator, we analyze the model problem depicted in Figure 2. This model problem couples two subdomains: a diskshaped bounded subdomain $\Omega_{1}$ of radius $R_{0}$ and an unbounded domain $\Omega_{0}=\mathbb{R}^{2} \backslash \Omega_{1}$ :

$$
\Omega_{0}:=\left\{\mathbf{x} \in \mathbb{R}^{2},|\mathbf{x}|>R_{0}\right\}, \quad \Omega_{1}:=\left\{\mathbf{x} \in \mathbb{R}^{2},|\mathbf{x}|<R_{0}\right\},
$$

with $\partial \Omega_{0}=\partial \Omega_{1}=\Sigma$. We study the spectral properties of the iteration operator obtained from the domain decomposition algorithm coupling these two subdomains. Studying the coupling of bounded and unbounded subdomains will allow us to understand the main properties that one could not analyze by 


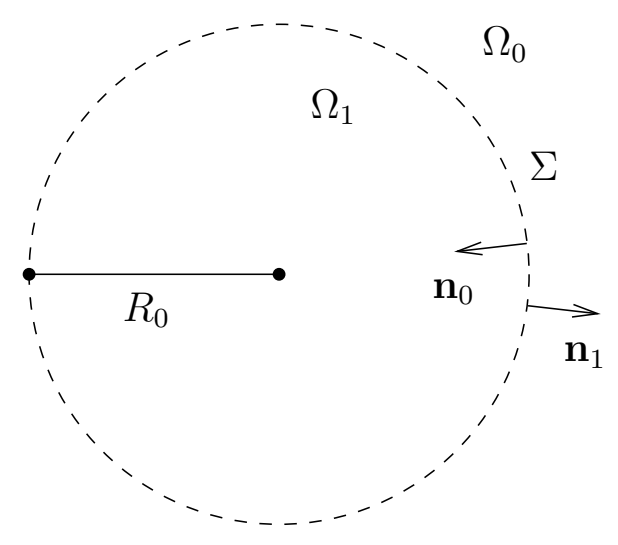

Figure 2: Model problem with two subdomains and a circular interface.

considering two bounded (e.g. a square domain divided in two) or two unbounded (e.g. two half-planes) subdomains. The considered model problem essentially contains the main difficulties encountered when solving exterior scattering problems. Note that a closely related problem where the unbounded domain is replaced by an annulus-shaped bounded domain with an ABC on the exterior boundary could also have been selected. However, this would only lead to more complex expressions without providing additional insight.

\subsection{Convergence Theorem}

For the considered model problem, the domain decomposition method with square-root transmission condition consists in solving separately at each iteration the following problems

$$
\left\{\begin{array}{l}
\Delta u_{0}+k^{2} u_{0}=0 \quad \text { in } \Omega_{0} \\
\partial_{\mathbf{n}_{0}} u_{0}+\mathcal{S}^{\mathrm{sq}, \varepsilon} u_{0}=g_{0} \quad \text { on } \Sigma \\
\lim _{|\mathbf{x}| \rightarrow \infty}|\mathbf{x}|^{1 / 2}\left(\partial_{|\mathbf{x}|} u_{0}-\imath k u_{0}\right)=0
\end{array}\right.
$$

and

$$
\left\{\begin{array}{l}
\Delta u_{1}+k^{2} u_{1}=0 \text { in } \Omega_{1}, \\
\partial_{\mathbf{n}_{1}} u_{1}+\mathcal{S}^{\mathrm{sq}, \varepsilon} u_{1}=g_{1} \text { on } \Sigma,
\end{array}\right.
$$

where $g_{0}$ and $g_{1}$ are defined as in equation (4). The convergence analysis can be developed by studying the spectral properties of the iteration operator $\mathcal{A}$ defined by (5), where

$$
\mathcal{A}:=\Pi \mathcal{T},
$$

and where the two operators $\mathcal{T}$ and $\Pi$ are defined as [15]:

$$
\mathcal{T} g^{(n)}:=\left(\begin{array}{cc}
\mathcal{T}_{1} & 0 \\
0 & \mathcal{T}_{0}
\end{array}\right)\left(\begin{array}{c}
g_{1}^{(n)} \\
g_{0}^{(n)}
\end{array}\right) \quad \text { and } \quad \Pi:=\left(\begin{array}{cc}
0 & I \\
I & 0
\end{array}\right),
$$

with

$$
\mathcal{T}_{1} g_{1}^{(n)}:=-g_{1}^{(n)}+2 \mathcal{S}^{\mathrm{sq}, \varepsilon} u_{1}^{(n+1)}, \quad \mathcal{T}_{0} g_{0}^{(n)}:=-g_{0}^{(n)}+2 \mathcal{S}^{\mathrm{sq}, \varepsilon} u_{0}^{(n+1)} .
$$


Since we have a circular geometry, we can analyze the error mode-by-mode by using a Fourier-Hankel series expansion in the polar coordinates system $(r, \theta)$. Let us set

$$
u_{\ell}(r, \theta)=\sum_{m=-\infty}^{+\infty} u_{\ell, m}(r) e^{\imath m \theta} \quad \text { and } \quad g_{\ell}(r, \theta)=\sum_{m=-\infty}^{+\infty} g_{\ell, m}(r) e^{\imath m \theta}, \quad \ell=0,1 .
$$

Since $\left\{e^{\imath m \theta}\right\}_{m \in \mathbb{Z}}$ defines an orthonormal basis of $L^{2}(\Sigma)$, problems (14) and (15) lead to solve the decoupled problems

$$
\left\{\begin{array}{l}
\frac{1}{r} \partial_{r}\left(r \partial_{r} u_{0, m}\right)-\frac{m^{2}}{r^{2}} u_{0, m}+k^{2} u_{0, m}=0 \text { for } r>R_{0}, \\
-\partial_{r} u_{0, m}+\mathcal{S}_{m}^{\mathrm{sq}, \varepsilon} u_{0, m}=g_{0, m} \quad \text { for } r=R_{0} \\
\lim _{r \rightarrow+\infty} r^{1 / 2}\left(\partial_{r} u_{0, m}-\imath k u_{0, m}\right)=0
\end{array}\right.
$$

and

$$
\left\{\begin{array}{l}
\frac{1}{r} \partial_{r}\left(r \partial_{r} u_{1, m}\right)-\frac{m^{2}}{r^{2}} u_{1, m}+k^{2} u_{1, m}=0 \quad \text { for } r<R_{0}, \\
\partial_{r} u_{1, m}+\mathcal{S}_{m}^{\mathrm{sq}, \varepsilon} u_{1, m}=g_{1, m} \quad \text { for } r=R_{0} .
\end{array}\right.
$$

In the polar coordinate system the surface divergence and the tangential gradient appearing in (10) reduce to the curvilinear derivative $R_{0}^{-1} \partial_{\theta}$. Hence, for a mode $m$, we get the analytical expression of the square-root transmission operator:

$$
\mathcal{S}_{m}^{\mathrm{sq}, \varepsilon}=-\imath k \sqrt{1-\frac{m^{2}}{k_{\varepsilon}^{2} R_{0}^{2}}}:=-\imath k\left(\mathcal{R}_{m}+\imath \mathcal{X}_{m}\right)
$$

The solution of the first and last equation of the exterior problem (20) is given by $u_{0, m}(r)=$ $\alpha_{m} H_{m}^{(1)}(k r)$, where $H_{m}^{(1)}$ denotes the Hankel function of the first kind and order $m$. For the interior problem (21) the solution of the first equation reads $u_{m}^{(1)}(r)=\beta_{m} J_{m}(k r)$, where $J_{m}$ is the Bessel function of order $m$.

Writing the modal decomposition of the operators $\mathcal{T}_{0}$ and $\mathcal{T}_{1}$ as $\mathcal{T}_{0}=\sum_{m=-\infty}^{+\infty} \mathcal{T}_{0, m} e^{\imath m \theta}$ and $\mathcal{T}_{1}=$ $\sum_{m=-\infty}^{+\infty} \mathcal{T}_{1, m} e^{\imath m \theta}$, one can show using the second equations of (20) and (21) that:

$$
\mathcal{T}_{\ell, m}=\frac{-k \mathcal{Z}_{\ell, m}+\mathcal{S}_{m}^{\mathrm{sq}, \varepsilon}}{k \mathcal{Z}_{\ell, m}+\mathcal{S}_{m}^{\mathrm{sq}, \varepsilon}}=\frac{-\mathcal{Z}_{\ell, m}+\imath\left(\mathcal{R}_{m}+\imath \mathcal{X}_{m}\right)}{\mathcal{Z}_{\ell, m}+\imath\left(\mathcal{R}_{m}+\imath \mathcal{X}_{m}\right)}, \quad \ell=0,1,
$$

with

$$
\mathcal{Z}_{0, m}=\frac{H_{m}^{(1)^{\prime}}\left(k R_{0}\right)}{H_{m}^{(1)}\left(k R_{0}\right)} \quad \text { and } \quad \mathcal{Z}_{1, m}=-\frac{J_{m}^{\prime}\left(k R_{0}\right)}{J_{m}\left(k R_{0}\right)} .
$$

Since the convergence properties of the DDM are strongly related to the spectrum of the iteration operator $\mathcal{A}$, we study the spectrum of the modal matrices

$$
\mathcal{A}_{m}=\left(\begin{array}{cc}
0 & \mathcal{T}_{0, m} \\
\mathcal{T}_{1, m} & 0
\end{array}\right)
$$

for each mode, where $\mathcal{A}=\sum_{m=-\infty}^{+\infty} \mathcal{A}_{m} e^{\imath m \theta}$. Let us begin by considering that the iteration equation (6) is solved using the Jacobi algorithm. Then, according to Theorem 1 below (see [15]), convergence occurs if and only if the spectral radius $\rho(\mathcal{A})=\max _{m \in \mathbb{Z}} \rho\left(\mathcal{A}_{m}\right)$ is smaller than one:

Theorem 1 Let $s \in \mathbb{R}$ be such that $\left(g_{0}, g_{1}\right) \in V:=H^{-s}(\Sigma) \times H^{-s}(\Sigma)$. The domain decomposition algorithm converges in $V$ if and only if for all $m, \rho\left(\mathcal{A}_{m}\right)<1$, with $\rho\left(\mathcal{A}_{m}\right)$ the largest modulus of the two eigenvalues of the $2 \times 2$ matrix $\mathcal{A}_{m}$. 
In our case, the two eigenvalues of $\mathcal{A}_{m}$ are: $\lambda_{m}^{ \pm}= \pm \sqrt{\mathcal{T}_{0, m} \mathcal{T}_{1, m}}$. Hence, it is sufficient to prove that $\left|\mathcal{T}_{0, m} \mathcal{T}_{1, m}\right|<1$ for each mode $m$ to guarantee the convergence of the iterative method. Before proving this inequality, let us first establish the following lemma.

Lemma 2 Consider the function $\mathcal{F}$ defined by

$$
\mathcal{F}(\mathcal{R}, \mathcal{X}, \mathcal{Z})=\frac{-\mathcal{Z}+\imath(\mathcal{R}+\imath \mathcal{X})}{\mathcal{Z}+\imath(\mathcal{R}+\imath \mathcal{X})},
$$

where the real coefficients $\mathcal{R}$ and $\mathcal{X}$ are positive $(\mathcal{R}>0, \mathcal{X}>0)$ and where $\mathcal{Z}$ is a complex number, with $\Re(\mathcal{Z}):=-x$ and $\Im(\mathcal{Z}):=y$. Then, under the conditions $x>0$ and $y>0$, we have

$$
|\mathcal{F}(\mathcal{R}, \mathcal{X}, \mathcal{Z})|<1
$$

Proof. By writing $\mathcal{Z}=-x+\imath y$, we obtain

$$
|\mathcal{F}(\mathcal{R}, \mathcal{X}, \mathcal{Z})|^{2}=\frac{(x-\mathcal{X})^{2}+(\mathcal{R}-y)^{2}}{(x+\mathcal{X})^{2}+(\mathcal{R}+y)^{2}}<1,
$$

since $(\mathcal{R}-y)^{2}<(\mathcal{R}+y)^{2}$ and $(x-\mathcal{X})^{2} \leq(x+\mathcal{X})^{2}$.

To prove the convergence of the algorithm, we first study separately the two coefficients $\mathcal{T}_{0, m}$ and $\mathcal{T}_{1, m}$, related to the exterior and interior problems respectively. The behavior of the coefficients depends on the choice of the damping parameter $\varepsilon$ appearing in the definition of the square-root operator (22) through (11).

Proposition 3 For $\varepsilon>0$ and any $m \in \mathbb{Z}$, we have:

$$
\left|\mathcal{T}_{0, m}\right|<1
$$

If $\varepsilon=0$ and $m \neq \pm k R_{0}$, then the inequality (29) also holds.

Proof. For any mode $m$, the coefficient $\mathcal{Z}_{0, m}$ can be written as

$$
\mathcal{Z}_{0, m}=\frac{H_{m}^{(1)^{\prime}}\left(k R_{0}\right) \overline{H_{m}^{(1)}}\left(k R_{0}\right)}{\left|H_{m}^{(1)}\left(k R_{0}\right)\right|^{2}}
$$

with $\Im\left(H_{m}^{(1)^{\prime}}\left(k R_{0}\right) \overline{H_{m}^{(1)}}\left(k R_{0}\right)\right)>0$ and $\Re\left(H_{m}^{(1)^{\prime}}\left(k R_{0}\right) \overline{H_{m}^{(1)}}\left(k R_{0}\right)\right)<0$ (see [15] for the proof).

Let us first assume that $\varepsilon=0$. If one has a propagating mode, i.e., $|m|<k R_{0}$, then $\mathcal{S}_{m}^{\text {sq, } 0}=-\imath k \mathcal{R}_{m}$. For the evanescent modes such that $k R_{0}<|m|, \mathcal{S}_{m}^{\text {sq, } 0}=k \mathcal{X}_{m}$. In these two cases, we deduce that $\left|\mathcal{T}_{0, m}\right|<1$ by using lemma 2 if there is no mode $m$ such that $m= \pm k R_{0}$.

Let us now consider the damped version $\varepsilon>0$ of the transmission operator $\mathcal{S}_{m}^{\mathrm{sq}, \varepsilon}$. We first recall that if $a$ and $b$ are two real numbers such that $b \neq 0$, then $\sqrt{a+\imath b}=p+\imath q$, where $p$ and $q$ are two real numbers given by

$$
p=\frac{1}{\sqrt{2}} \sqrt{\sqrt{a^{2}+b^{2}}+a}, \quad q=\frac{\operatorname{sign}(b)}{\sqrt{2}} \sqrt{\sqrt{a^{2}+b^{2}}-a} .
$$

Expanding $\mathcal{S}_{m}^{\mathrm{sq}, \varepsilon}=-\imath k\left(\mathcal{R}_{m}+\imath \mathcal{X}_{m}\right)$ as

$$
\mathcal{S}_{m}^{\mathrm{sq}, \varepsilon}=-\imath k \sqrt{\frac{R_{0}^{2}\left(\left(k^{2}-\varepsilon^{2}\right)^{2}+4 k^{2} \varepsilon^{2}\right)-m^{2}\left(k^{2}-\varepsilon^{2}\right)+2 \imath m^{2} k \varepsilon}{R_{0}^{2}\left(\left(k^{2}-\varepsilon^{2}\right)^{2}+4 k^{2} \varepsilon^{2}\right)}},
$$

and knowing that $k>0$ and $\varepsilon>0$, we get that $\mathcal{R}_{m}>0$ and $\mathcal{X}_{m}>0$. Using (31) and Lemma 2, this allows us to conclude that $\left|\mathcal{T}_{0, m}\right|<1$, for any $m \in \mathbb{Z}$ and $\varepsilon>0$.

Concerning the interior problem, we have the following result. 
Proposition 4 If $\varepsilon \geq 0$ and $|m|>k R_{0}$, we have

$$
\left|\mathcal{T}_{1, m}\right|<1
$$

If $\varepsilon=0$ and $|m| \leq k R_{0}$, we have

$$
\left|\mathcal{T}_{1, m}\right|=1
$$

Proof. We observe that $\mathcal{Z}_{1, m}$ is real. Let us first consider the case when $\varepsilon=0$. If $|m| \leq k R_{0}$, then $\mathcal{X}_{m}=0$ and we trivially have $\left|\mathcal{T}_{1, m}\right|=1$. When $|m|>k R_{0}$, we study the sign of $\mathcal{Z}_{1, m}$. Since

$$
\mathcal{Z}_{1, m}=-\frac{J_{m}^{\prime}\left(k R_{0}\right)}{J_{m}\left(k R_{0}\right)}=-\frac{u_{1, m}^{\prime}\left(R_{0}\right)}{k u_{1, m}\left(R_{0}\right)},
$$

by using the variational formulation of problem (21) we obtain

$$
\Re\left(R_{0} u_{1, m}^{\prime}\left(R_{0}\right) \overline{u_{1, m}}\left(R_{0}\right)\right)=\int_{0}^{R_{0}}\left\{r\left|u_{1, m}^{\prime}\right|^{2}+\left(\frac{m^{2}}{r^{2}}-k^{2}\right)\left|u_{1, m}\right|^{2} r\right\} d r .
$$

Hence, for all $|m|>k R_{0}$, we deduce that $\Re\left(R_{0} u_{1, m}^{\prime}\left(R_{0}\right) \overline{u_{1, m}}\left(R_{0}\right)\right)>0$. Furthermore, $\mathcal{R}_{m}=0$ but $\mathcal{X}_{m} \neq 0$, meaning that $\left|\mathcal{T}_{1, m}\right|<1$.

Let us now consider the case $\varepsilon>0$. If $|m|>k R_{0}$ the same procedure can be used, in which case $\mathcal{R}_{m}>0$ and $\left|\mathcal{T}_{1, m}\right|<1$

¿From the preceding propositions we can prove the following result.

Theorem 5 For $\varepsilon=0$ and if $m$ is not a cut-off mode, i.e. $|m| \neq k R_{0}$, we have the inequality

$$
\left|\mathcal{T}_{0, m} \mathcal{T}_{1, m}\right|<1 \text {. }
$$

If $\varepsilon>0$, then there exists $\varepsilon_{\max }$ such that for $0<\varepsilon<\varepsilon_{\max }$ the inequality (35) is also satisfied.

Proof. Let $\varepsilon=0$ and let us assume that there is no cut-off mode. Then, the result is a consequence of Propositions 3 and 4 . If $\varepsilon>0$, the inequality (35) is true if $m$ corresponds to an evanescent mode. However, we cannot conclude directly for the propagating ones because of the operator $\mathcal{T}_{1}$. These propagating modes are in finite number and for $\varepsilon=0$ we have (35). Therefore, we can then deduce by continuity on $\varepsilon$ that there exists $\varepsilon_{\max }$ such that for $0<\varepsilon<\varepsilon_{\max }$, the inequality (35) is satisfied.

The preceding theorem ensures convergence of the Jacobi domain decomposition algorithm with the square-root operator when $0<\varepsilon<\varepsilon_{\max }$. The analysis is not valid for the cut-off mode $|m|=k R_{0}$ when $\varepsilon=0$ : indeed, the operator $\mathcal{S}^{\mathrm{sq}, \varepsilon}$ is then not invertible, which makes the domain decomposition algorithm ill-posed. In the actual implementation of the DDM it is thus important to consider $\varepsilon>0$ in order to guarantee the well-posedness of the DDM. In addition, as we will show, $\varepsilon>0$ contributes in improving the convergence in the transition zone between the propagating and evanescent modes.

\subsection{Quasi-Optimality}

Since

$$
H_{m}^{(1)}(t) \approx-\frac{2^{m}(m-1) !}{i \pi t^{m}} \quad \text { and } \quad J_{m}(t) \approx-\frac{t^{m}}{2^{m} m !} \quad \text { for }|m| \gg|t|,
$$

we get, for $|m| \gg k R_{0}$,

$$
\mathcal{Z}_{0, m} \approx-\frac{m}{k R_{0}} \quad \text { and } \quad \mathcal{Z}_{1, m} \approx \frac{m}{k R_{0}}
$$

and thus

$$
\lim _{m \longrightarrow \infty} \lambda_{m}^{ \pm}= \pm \frac{\imath \varepsilon}{-2 k-\imath \varepsilon} \quad \text { for } \varepsilon>0
$$



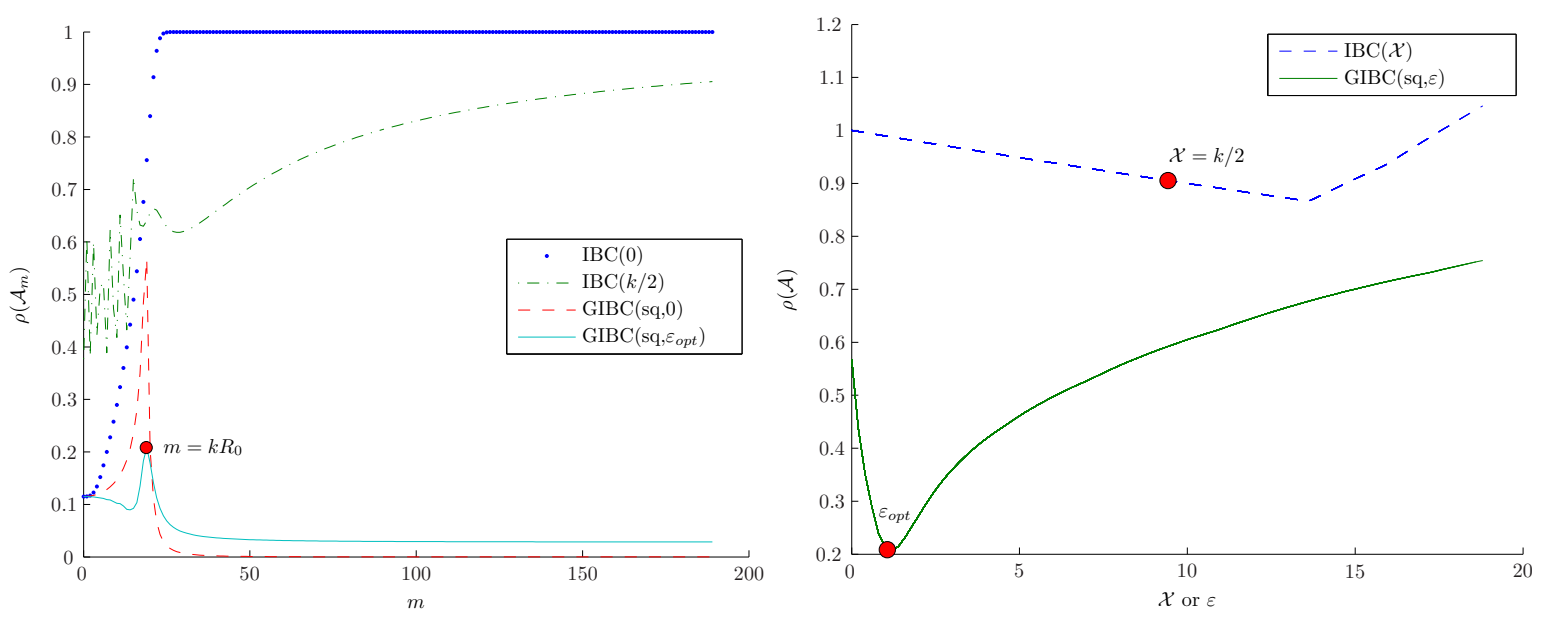

(a) Spectral radius of the modal iteration operator $\mathcal{A}_{m}$ vs. (b) Spectral radius of the iteration operator $\mathcal{A}$ vs. the the mode $m$.

damping parameter $\varepsilon$.

Figure 3: Spectral radius of the iteration operators and optimal choice of the damping coefficient $\varepsilon$.

This shows that there is a clustering of the eigenvalues for $|m| \gg k R_{0}$. Obviously, the limit is zero if $\varepsilon=0$. Therefore, the rate of convergence is optimal for the evanescent modes. This property remains true for $\varepsilon>0$ since $k \gg \varepsilon$.

To demonstrate the efficiency of the algorithm on the rest of the modes, let us choose $k=6 \pi, R_{0}=1$ and a maximal number of modes $m^{\max }=\left[10 k R_{0}\right]$ (where $\left[10 k R_{0}\right]$ denotes the integer part of $10 k R_{0}$ ), and let us compare the new algorithm with the Després and EMDA algorithms. We report in Figure 3(a) the modal spectral radius $\rho\left(\mathcal{A}_{m}\right)$ with respect to $m$ for the transmitting boundary conditions $\operatorname{IBC}(0)$ (Després), $\operatorname{IBC}(k / 2)$ (EMDA) and $\operatorname{GIBC}(\mathrm{sq}, 0)$. As is well-known, $\operatorname{IBC}(0)$ has a spectral radius equal to 1 for the evanescent modes, which is improved by $\operatorname{IBC}(k / 2)$. For the new transmission condition with $\varepsilon=0$, we clearly observe an optimal convergence rate for the evanescent part, which is coherent with our previous comment. We also observe a significant improvement over the Després and EMDA algorithms on the propagating modes.

The damping parameter $\varepsilon$ can be optimized to further improve the spectrum of the iteration operator corresponding to the modes in the transition zone. If we denote by $\rho^{\mathrm{sq}, \varepsilon}$ the spectral radius of $\mathcal{A}_{m}$ when the $\operatorname{GIBC}(\mathrm{sq}, \varepsilon)$ transmission condition is used, we look for $\varepsilon_{\mathrm{opt}}$ such that

$$
\rho^{\mathrm{sq}, \varepsilon_{\mathrm{opt}}}=\min _{\varepsilon>0} \rho^{\mathrm{sq}, \varepsilon} .
$$

As mentioned above, the square-root operator is a suitable approximation of the DtN operator for the propagating and the evanescent modes. By construction, it appears that the maximal error between the exact DtN operator and its square-root approximation $\mathcal{S}^{\mathrm{sq}, \varepsilon}$ is obtained for the modes $m$ satisfying $m= \pm\left[k R_{0}\right][6]$. These specific modes are better known as grazing modes in the context of scattering problems. For such problems it was shown in [6] that the optimal value of the damping parameter is $\varepsilon_{\mathrm{opt}}=0.4 k^{1 / 3} \mathcal{H}^{2 / 3}$, where $\mathcal{H}$ is the mean curvature on $\Sigma$. The resulting modal spectral radius for $\operatorname{GIBC}\left(\mathrm{sq}, \varepsilon_{\mathrm{opt}}\right)$ is shown in Figure 3(a), where the improvement around $m=k R_{0}$ is clearly visible. We can see in Figure 3(b) that the spectral radius of the iteration operator is indeed minimum for $\varepsilon=\varepsilon_{\mathrm{opt}}$. From Figure 3(b) one can also observe that $\mathcal{X}_{\text {opt }}=k / 2$ (the value chosen for EMDA in [15, 16]) is a fairly good choice to minimize the spectral radius for $\operatorname{IBC}(\mathcal{X})$. In this example, $\mathcal{X}_{\text {opt }}=3 k / 4$ could also be a good choice. 


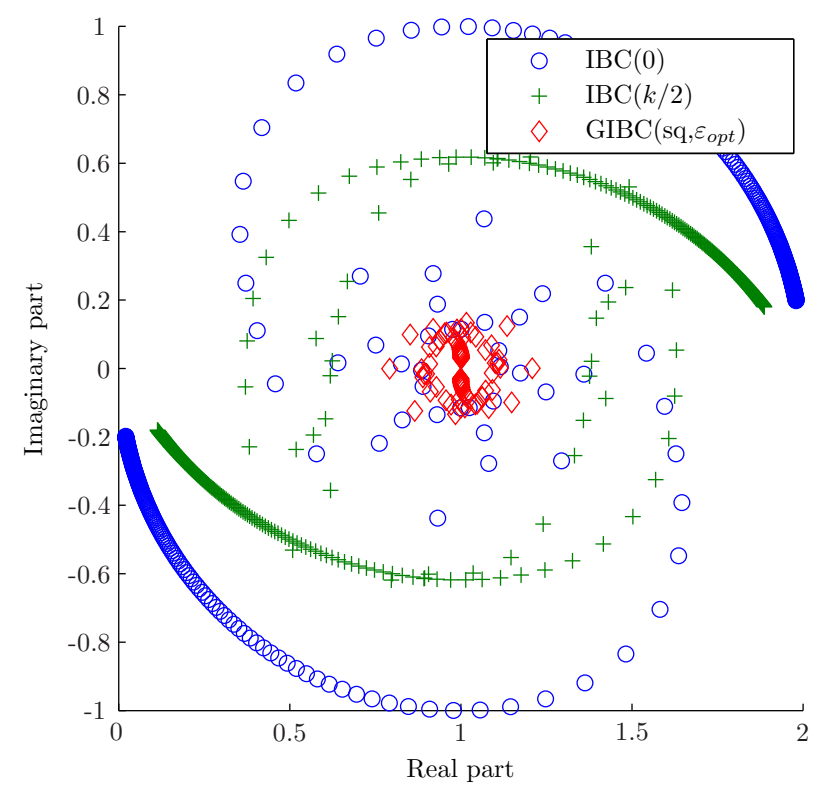

Figure 4: Eigenvalues distribution in the complex plane for $(I-\mathcal{A})$ and different transmission operators.

\subsection{Krylov Subspace Solvers}

Now, let us analyze the solution of (6) by using a Krylov subspace method like GMRES. It is well-known that fast convergence of the GMRES solver is related to the existence of clustering of its eigenvalues. Let us recall that the equation that we solve is given by the operator: $(I-\mathcal{A})$. Therefore, its eigenvalues are: $\mu_{m}^{ \pm}=1 \mp \lambda_{m}^{ \pm}$for each mode $m \in \mathbb{Z}$. We report in Figure 4 the spectrum of the iteration operator for $\operatorname{IBC}(0), \operatorname{IBC}(k / 2)$ and $\operatorname{GIBC}\left(\mathrm{sq}, \varepsilon_{\mathrm{opt}}\right)$. We consider again $k R_{0}=6 \pi$ for a maximal number of modes $m^{\max }=\left[10 k R_{0}\right]$. For all transmission operators the spectrum lies in the right half-plane, which makes the GMRES converging. However, for $\operatorname{IBC}(0)$ many eigenvalues spread out in the complex plane. A slightly better clustering occurs for $\operatorname{IBC}(k / 2)$, while there is an excellent clustering of the eigenvalues for GIBC(sq, $\left.\varepsilon_{\mathrm{opt}}\right)$. In particular, only a few eigenvalues related to the propagating modes do not cluster but are very close to $(1,0)$. Furthermore, the eigenvalues related to the evanescent modes seem to cluster at $(1,0)$. According to $(38)$, we show that the clustering is at point

$$
C^{*}=\frac{k+\imath \varepsilon_{\mathrm{opt}}}{k+\imath \varepsilon_{\mathrm{opt}} / 2}
$$

(in our case $C^{*}=1.0008+0.0282 \imath$ ). Since the eigenvalues cluster for the evanescent modes, we can expect that in the numerical implementation the GMRES convergence rate will be independent of the density of discretization points per wavelength $n_{\lambda}[3,4,5]$. This will be indeed the case as seen in Section 7 .

\section{Localization of the Square-Root Operator by Complex Padé Approximants}

The square-root operator (10) is a nonlocal operator (a pseudo-differential operator of order +1 ). Therefore, it is impractical in a finite element setting since it would lead to consider full matrices for the transmission boundaries. Fortunately, a localization process of this operator can be efficiently done by 

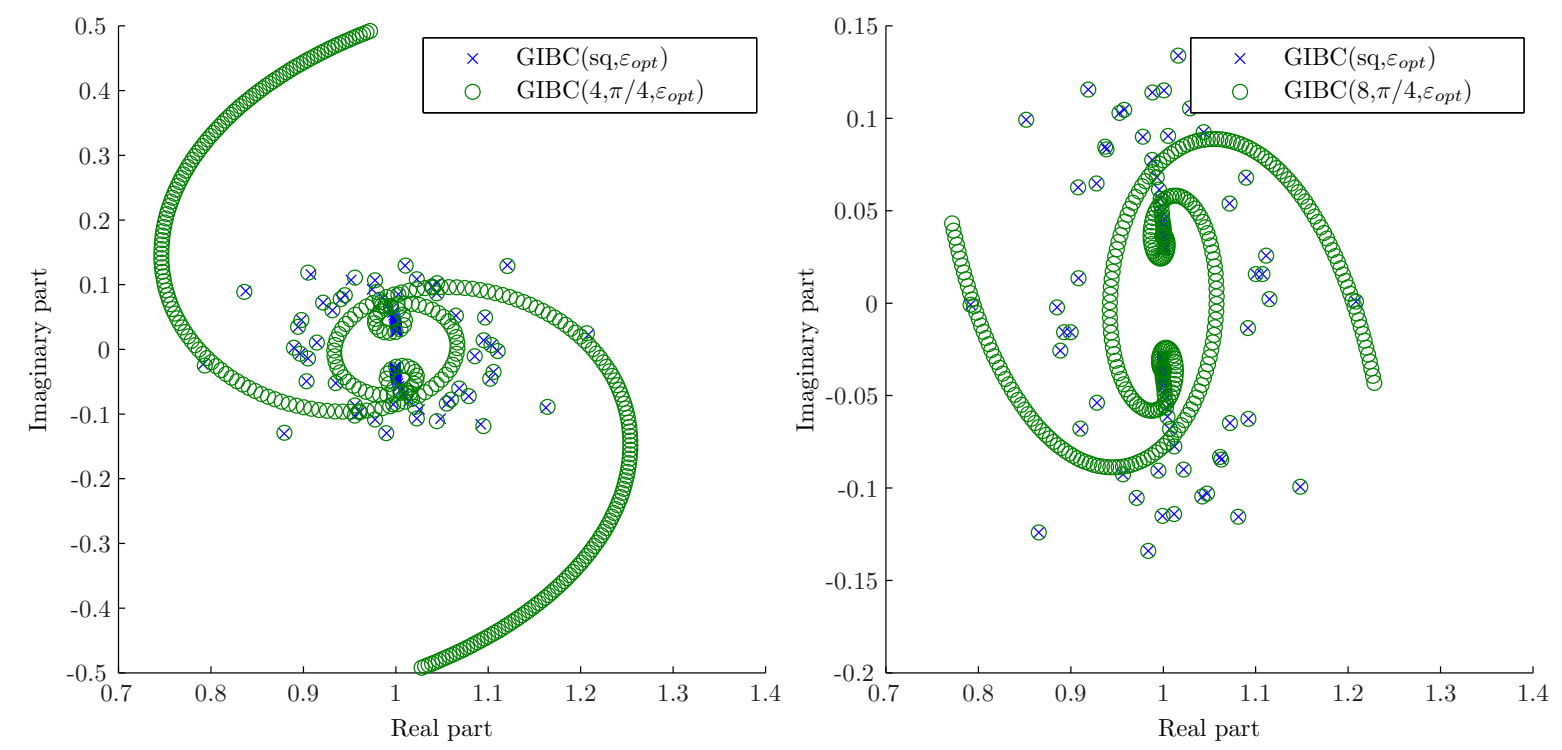

Figure 5: Eigenvalue distribution in the complex plane for the exact and Padé-localized square-root transmission operator of order 4 (left) and 8 (right).

using partial differential (local) operators to have a sparse matrix representation. In $[35,34,6]$, this is realized by a rotating branch-cut approximation of the square-root and then applying complex Padé approximants of order $N_{p}[38]$ :

$$
\begin{aligned}
\left.\sqrt{1+\operatorname{div}_{\Gamma^{\infty}}(} \frac{1}{k_{\varepsilon}^{2}} \nabla_{\Gamma^{\infty}}\right) u & \approx R_{N_{p}}^{\alpha}\left(\operatorname{div}_{\Gamma^{\infty}}\left(\frac{1}{k_{\varepsilon}^{2}} \nabla_{\Gamma^{\infty}}\right)\right) u \\
& =C_{0} u+\sum_{\ell=1}^{N_{p}} A_{\ell} \operatorname{div}_{\Gamma^{\infty}}\left(k_{\varepsilon}^{-2} \nabla_{\Gamma^{\infty}}\right)\left(1+B_{\ell} \operatorname{div}_{\Gamma^{\infty}}\left(k_{\varepsilon}^{-2} \nabla_{\Gamma^{\infty}}\right)\right)^{-1} u,
\end{aligned}
$$

which corresponds to the complex Padé approximation

$$
\sqrt{1+z} \approx R_{N_{p}}^{\alpha}(z)=C_{0}+\sum_{\ell=1}^{N_{p}} \frac{A_{\ell} z}{1+B_{\ell} z} .
$$

Let us mention some similar ideas in the approximation of the square-root with the approach described in [29]. The complex coefficients $C_{0}, A_{\ell}$ and $B_{\ell}$ are given by

$$
C_{0}=e^{\imath \frac{\alpha}{2}} R_{N_{p}}\left(e^{-\imath \alpha}-1\right), A_{\ell}=\frac{e^{-\frac{\imath \alpha}{2}} a_{\ell}}{\left(1+b_{\ell}\left(e^{-\imath \alpha}-1\right)\right)^{2}}, B_{\ell}=\frac{e^{-\imath \alpha} b_{\ell}}{1+b_{\ell}\left(e^{-\imath \alpha}-1\right)} .
$$

In $(43) \alpha$ is the angle of rotation, $\left(a_{\ell}, b_{\ell}\right), \ell=1, \ldots, N_{p}$, are the standard real Padé coefficients

$$
a_{\ell}=\frac{2}{2 N_{p}+1} \sin ^{2}\left(\frac{\ell \pi}{2 N_{p}+1}\right), b_{\ell}=\cos ^{2}\left(\frac{\ell \pi}{2 N_{p}+1}\right),
$$

and $R_{N_{p}}$ is the real Padé approximant of order $N$ :

$$
\sqrt{1+z} \approx R_{N_{p}}(z)=1+\sum_{\ell=1}^{N_{p}} \frac{a_{\ell} z}{1+b_{\ell} z} .
$$


For a variational representation, the approximation of the Padé-localized square-root transmission operator (10)-(45) is realized by using auxiliary coupled functions $[35,34,6]$

$$
S^{N_{p}, \alpha, \varepsilon} u=-\imath k\left(C_{0} u+\sum_{\ell=1}^{N_{p}} A_{\ell} \operatorname{div}_{\Gamma^{\infty}}\left(\frac{1}{k_{\varepsilon}^{2}} \nabla_{\Gamma^{\infty}} \varphi_{\ell}\right)\right) \quad \text { on } \Gamma^{\infty},
$$

where the functions $\varphi_{\ell}, \ell=1, . ., N_{p}$, are defined on $\Gamma^{\infty}$ as the solutions of the following surface PDEs:

$$
\left(1+B_{\ell} \operatorname{div}_{\Gamma^{\infty}}\left(\frac{1}{k_{\varepsilon}^{2}} \nabla_{\Gamma^{\infty}}\right)\right) \varphi_{\ell}=u .
$$

The resulting transmitting boundary condition is again a Generalized Impedance Boundary Condition, and is denoted by $\operatorname{GIBC}\left(N_{p}, \alpha, \varepsilon\right)$ for the Padé approximation with $N_{p}$ auxiliary functions, for an angle of rotation $\alpha$ and a damping parameter $\varepsilon$. The lowest-order approximation would be $S^{N_{p}, \alpha, \varepsilon}=-\imath k I$ which also corresponds to the original approximation $\mathcal{S}^{0}$ of $\mathcal{S}$, i.e., $\operatorname{IBC}(0)$.

Let us analyze on the model problem of Section 4 the effect of the Padé approximation on the rate of convergence. For a mode $m$, the transmission operator acts as

$$
\mathcal{S}_{m}^{N_{p}, \alpha, \varepsilon}=-\imath k R_{N_{p}}^{\alpha}\left(-\frac{m^{2}}{k_{\varepsilon}^{2} R_{0}^{2}}\right) .
$$

In the following the angle of rotation $\alpha$ is always taken equal to $\pi / 4$, which was found to be the optimal choice through numerical experiments. We report in Figure 5 the spectrum of GIBC(sq, $\left.\varepsilon_{\mathrm{opt}}\right)$, $\operatorname{GIBC}\left(4, \pi / 4, \varepsilon_{\mathrm{opt}}\right)$ and $\operatorname{GIBC}\left(8, \pi / 4, \varepsilon_{\mathrm{opt}}\right)$. As previously noticed, there is an excellent clustering of the eigenvalues for GIBC(sq, $\varepsilon_{\text {opt }}$ ). As expected, the larger $N_{p}$, the better the approximation of the spectrum of the square-root. Moreover, $N_{p}$ allows to adjust the spectrum accuracy for large modes $m$ (evanescent modes). Hence, it can be conjectured that $N_{p}$ must be taken with respect to the density of discretization points per wavelength $n_{\lambda}$. We will see in the numerical simulations (see Section 7 ) that relatively small values of $N_{p}\left(N_{p}=2,4,8\right)$ give optimal convergence results.

\section{Finite Element Implementation}

Let us now describe the implementation of the domain decomposition algorithm with Padé-type transmission condition in a finite element context. Recall first that the iterative method consists in solving problems (3) and computing the transmitted quantities (4). We restrict this description only to a problem posed in a domain, noted here $\Omega$, with no exterior interface nor interface connected to the scatterer. This simply implies that $\Gamma_{i}=\emptyset, \Gamma_{i}^{\infty}=\emptyset$ and $\partial \Omega$ is an artificial interface. The extention to the other cases

is direct. For the sake of clarity, we abbreviate the notations $u_{i}^{(n+1)}, g_{i j}^{(n)}$ and $g_{i j}^{(n+1)}$, used in problem (3) and equation (4), by $u, g_{\text {in }}$ and $g_{\text {out }}$ respectively. An iteration then leads to solving first the interior boundary value problem in terms of $u$ with coupling auxiliary functions $\left\{\varphi_{\ell}\right\}_{\ell=1, \ldots, N_{p}}$ according to the use of Padé approximation of order $N_{p}$ :

$$
\left\{\begin{array}{l}
\Delta u+k^{2} u=0 \quad \text { in } \Omega, \\
\partial_{\mathbf{n}} u-\imath k C_{0} u-\imath k \sum_{\ell=1}^{N_{p}} A_{\ell} \operatorname{div} \partial \Omega\left(\frac{1}{k_{\varepsilon}^{2}} \nabla_{\partial \Omega} \varphi_{\ell}\right)=g_{\text {in }} \quad \text { on } \partial \Omega, \\
-u+B_{\ell} \operatorname{div}_{\partial \Omega}\left(\frac{1}{k_{\varepsilon}^{2}} \nabla_{\partial \Omega} \varphi_{\ell}\right)+\varphi_{\ell}=0, \ell=1, \ldots, N_{p}, \quad \text { on } \partial \Omega,
\end{array}\right.
$$

where $C_{0}, A_{\ell}$ and $B_{\ell}$ are given by (43). The auxiliary functions $\left\{\varphi_{\ell}\right\}_{\ell=1, \ldots, N_{p}}$ are introduced to deal with the use of the Padé approximation of order $N_{p}$. Considering now test-functions $v \in H^{1}(\Omega)$ and $v_{\ell} \in H^{1}(\partial \Omega), \ell=1, \ldots, N_{p}$, we get the coupled variational formulation 


$$
\left\{\begin{array}{c}
\int_{\Omega_{i}}\left(\nabla u \cdot \nabla v-k^{2} u v\right) d \Omega-\imath k C_{0} \int_{\partial \Omega} u v d \partial \Omega \\
+\imath k \sum_{\ell=1}^{N_{p}} A_{\ell} \int_{\partial \Omega} \frac{1}{k_{\varepsilon}^{2}} \nabla_{\partial \Omega} \varphi_{\ell} \cdot \nabla_{\partial \Omega} v d \partial \Omega=-\int_{\partial \Omega} g_{\text {in }} v d \partial \Omega \\
-\int_{\partial \Omega} u v_{\ell} d \partial \Omega-B_{\ell} \int_{\partial \Omega} \frac{1}{k_{\varepsilon}^{2}} \nabla_{\partial \Omega} \varphi_{\ell} \cdot \nabla_{\partial \Omega} v_{\ell} d \partial \Omega \\
+\int_{\partial \Omega} \varphi_{\ell} v_{\ell} d \partial \Omega=0, \ell=1, \ldots, N_{p}
\end{array}\right.
$$

Following the solution of (50), we update the boundary data through the relation

$$
g_{\text {out }}=-g_{\text {in }}-2 \imath k C_{0} u-2 \imath k \sum_{\ell=1}^{N_{p}} A_{\ell} \operatorname{div} \partial \Omega\left(\frac{1}{k_{\varepsilon}^{2}} \nabla_{\partial \Omega} \varphi_{\ell}\right), \quad \text { on } \partial \Omega .
$$

Let us now consider a covering $\Omega_{h}$ of $\Omega$ using $n_{t}$ tetrahedral (or triangular in two-dimensions) finite elements with $n_{v}$ vertices. Parameter $h$ is the usual finite element notation for the maximal side length of the tetrahedrals. All the notations are extended with $h$ as subscript for the discrete version of the domains as well as unknowns. We choose here linear finite element approximations but the extension to higher-order elements is straightforward. For scattering problems, it is common to introduce the density of discretization points per wavelength: $n_{\lambda}=\lambda / h$. The local density of discretization points is therefore: $n_{\lambda}=\lambda / h$. Let us denote by $\mathbb{S}^{\Omega_{h}}$ and $\mathbb{M}^{\Omega_{h}}$ respectively the stiffness and mass matrices for linear elements associated with the domains $\Omega_{h}$. The matrices have size $n_{v} \times n_{v}$. Furthermore, we introduce $\mathbb{S}^{\partial \Omega_{h}}$ and $\mathbb{M}^{\partial \Omega_{h}}$ as the respective stiffness and mass matrices related to the transmitting surface $\partial \Omega_{h}$. If these correspond to a generalized stiffness matrix for a surface function $\beta$, then it is quoted $\mathbb{S}_{\beta}^{\partial \Omega_{h}}$. All these matrices have a size $n_{t}^{\partial} \times n_{t}^{\partial}$. Let us denote by $\mathbf{u} \in \mathbb{C}^{n_{v}}$ the local unknown vector and $\varphi_{\ell} \in \mathbb{C}^{n_{t}^{\partial}}$ the surface unknown auxiliary vectors obtained with linear finite elements. The discrete test-vectors and right hand side are also bold typed. Then, the discretization of the variational problem (49) leads to the solution of the coupled linear system

$$
\left\{\begin{array}{l}
\left(\mathbb{S}^{\Omega_{h}}-k^{2} \mathbb{M}^{\Omega_{h}}-\imath k C_{0} \mathbb{M}^{\partial \Omega_{h}}\right) \mathbf{u}+\imath k \sum_{\ell=1}^{N_{p}} A_{\ell} \mathbb{S}_{k_{\varepsilon}^{-2}}^{\partial \Omega_{h}} \boldsymbol{\varphi}_{\ell}=-\mathbb{M}^{\partial \Omega_{h}} \mathbf{g}_{\mathrm{in}} \\
-\mathbb{M}^{\partial \Omega_{h}} \mathbf{u}-\left(B_{\ell} \mathbb{S}_{k_{\varepsilon}^{-2}}^{\partial \Omega_{h}}-\mathbb{M}^{\partial \Omega_{h}}\right) \boldsymbol{\varphi}_{\ell}=\mathbf{0}, \quad \ell=1, \ldots, N_{p}
\end{array}\right.
$$

The size of this linear system is $\left(n_{v}+\left(1+N_{p}\right) n_{t}^{\partial}\right) \times\left(n_{v}+\left(1+N_{p}\right) n_{t}^{\partial}\right)$. Each augmented system is solved directly by a LU factorization method since each matrix has a moderate size. The boundary data update can be done by vector addition using relation (4).

\section{$7 \quad$ Numerical Results}

This section is devoted to numerical simulations validating the new domain decomposition algorithm using the Padé-localized square-root transmission condition. We use the finite element method with linear (P1) basis functions implemented in GetDP/Gmsh [22,28] to approximate the local problems (3), as explained in Section 6. The convergence criterion for all the presented examples is identical: the iterations are stopped when the initial residual has decreased by a factor of $10^{-6}$.

\subsection{Two-Dimensional Examples}

The two-dimensional examples concern the scattering of a plane wave by a unit sound-soft circular cylinder. The second-order Bayliss-Turkel $[9,2,10]$ artificial boundary condition is set on a fictitious 


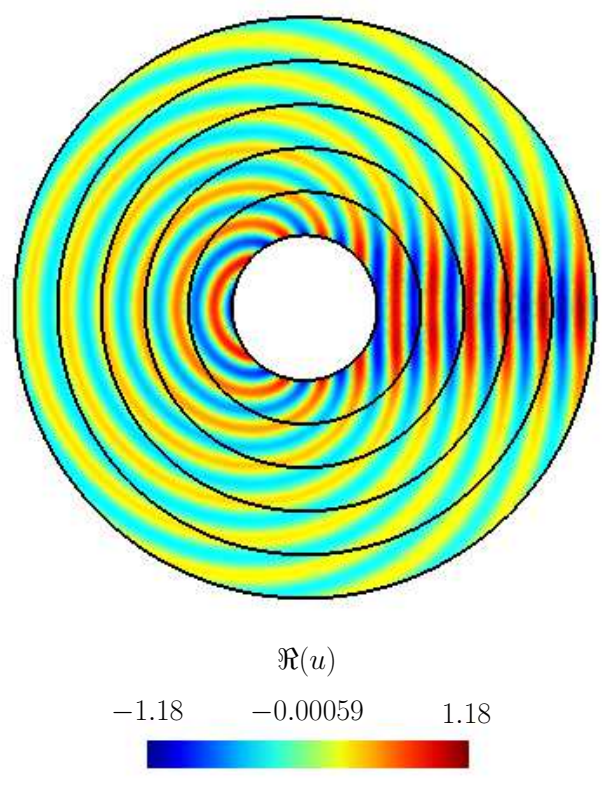

(a) "circle-concentric" decomposition

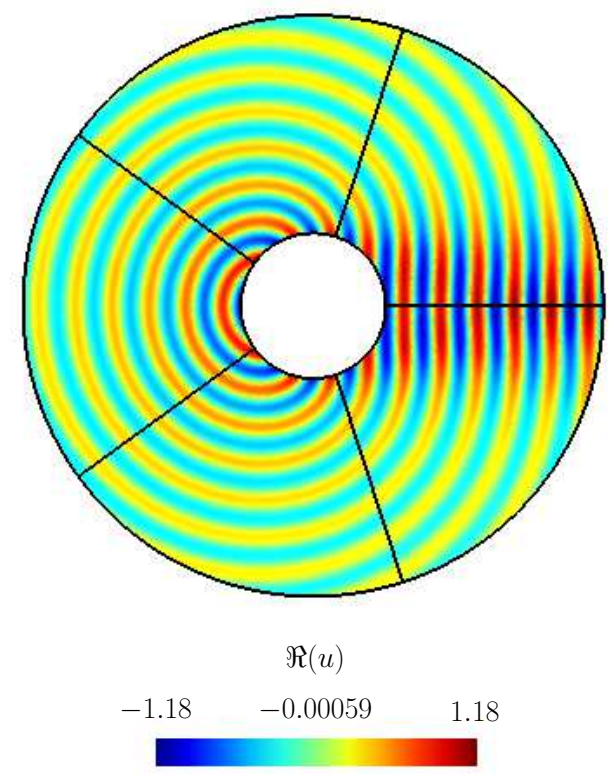

(b) "circle-pie" decomposition

Figure 6: Two-dimensional test cases: reconstruction of the scattered field on the global domain after a DDM computation with $k=4 \pi$ and $N_{\text {dom }}=5$. 


\begin{tabular}{|c|c|c|}
\hline$N_{\text {dom }}$ & Jacobi & GMRES \\
\hline 2 & 12 & 9 \\
\hline 5 & 45 & 24 \\
\hline 10 & 152 & 52 \\
\hline 15 & +500 & 74 \\
\hline
\end{tabular}

\begin{tabular}{|c|c|c|}
\hline$k$ & Jacobi & GMRES \\
\hline$\pi$ & 102 & 41 \\
\hline $2 \pi$ & 62 & 36 \\
\hline $3 \pi$ & 52 & 38 \\
\hline $4 \pi$ & 64 & 38 \\
\hline
\end{tabular}

Table 1: Number of iterations vs. number of subdomains for $k=\pi$ (left) and Number of iterations vs. wavenumber for $N_{\text {dom }}=8$ (right) when using the Jacobi or GMRES algorithm, for the "circle-concentric" decomposition.

circular boundary with radius 4 (see Figure 6).

\subsubsection{Jacobi vs. GMRES}

We begin by analyzing the behavior of the iterative methods (successive approximations, i.e. Jacobi, or GMRES [42]) when the domain is partitioned into $N_{\text {dom }}$ concentric subdomains. We call this partitioning the "circle-concentric" decomposition (see Figure 6(a)). The "size" $L_{i}$ of the subdomain $\Omega_{i}$, defined as the difference between its exterior and interior radius, is equal to $3 / N_{\text {dom }}$.

Le us start by considering a wavenumber $k=\pi$ and a density of discretization points per wavelength $n_{\lambda}=12$. We report in Table 1 (left) the number of iterations required using Jacobi and GMRES. Concerning the complex Padé approximation, we fix $N_{p}=8$ auxiliary equations and $\theta=\pi / 4$. The damping parameter $\varepsilon$ is optimized according to $\varepsilon_{\mathrm{opt}}=0.6 k^{1 / 3} R_{j}^{-2 / 3}$ (where $R_{j}$ is the radius of the $j$-th interface). Let us precise that the "size" of the overall domain is $L=3=1.5 \lambda$. This means that in the case of $N_{\text {dom }}=10$ (resp. $N_{\text {dom }}=15$ ) subdomains, the size of one subdomain is about $0.15 \lambda$ (resp. about $0.1 \lambda$ ) which is very small. For these cases, the EMDA algorithm solved by the Jacobi method diverges while our approach converges. As it can be seen, the algorithm scales according to $N_{\text {dom }}$, and as expected, the GMRES outperforms the Jacobi method.

Consider now a partition of the initial domain into $N_{\mathrm{dom}}=8$. The discretization is again fixed by $n_{\lambda}=12$. The same parameters $\left(N_{p}=8, \theta=\pi / 4\right.$ and $\left.\varepsilon_{\mathrm{opt}}=0.6 k^{1 / 3} R_{j}^{-2 / 3}\right)$ are kept to approximate the transmission operator given by (41). We report in Table 1 (right) the number of iterations for converging according to the wavenumber $k$. As we can see, the convergence is quasi independent of the wavenumber $k$. This is known to be a very difficult goal to attain when designing iterative schemes for scattering problems. In particular, this means that our Padé-type transmission condition with GMRES DDM is well-suited for high frequency scattering problems - we will analyze this behaviour further in Section 7.1.2.

¿From these first numerical results we clearly see that the GMRES algorithm outperforms the successive approximations (Jacobi), especially when the number of subdomains is large. In what follows we will thus only report results for the GMRES algorithm.

\subsubsection{Influence of the Wavenumber, the Discretization Density and the Number of Sub- domains}

Let us consider $N_{\text {dom }}=5$ subdomains. Figure 7 (a) shows the number of iterations to reach convergence with respect to the wavenumber $k$, for two densities of discretization points per wavelength $n_{\lambda}$. For EMDA with $\operatorname{IBC}(k / 2)$, the number of iterations increases with respect to $k$ and $n_{\lambda}$. Concerning $\operatorname{GIBC}\left(N_{p}, \pi / 4\right.$, $\left.\varepsilon_{\text {opt }}\right)$, we can see that the convergence rate is independent of both the wavenumber and density of discretization points per wavelength. Furthermore, in this case, $N_{p}=2$ provides already an optimal convergence compared with $N_{p}=8$. Let us recall that one of the important points here is that we use complex approximants. Using real Padé approximants would lead to much higher values of $N_{p}$, hence 


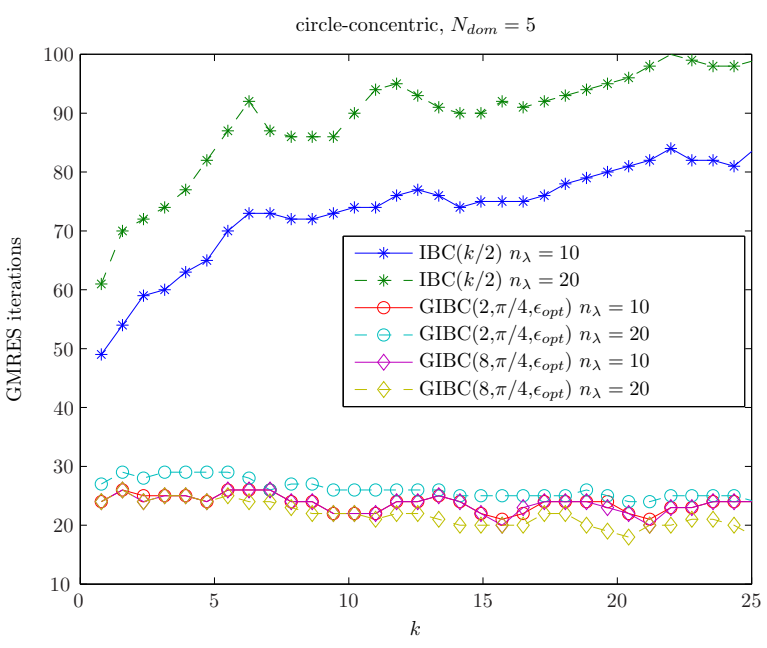

(a) Number of iterations vs. wavenumber

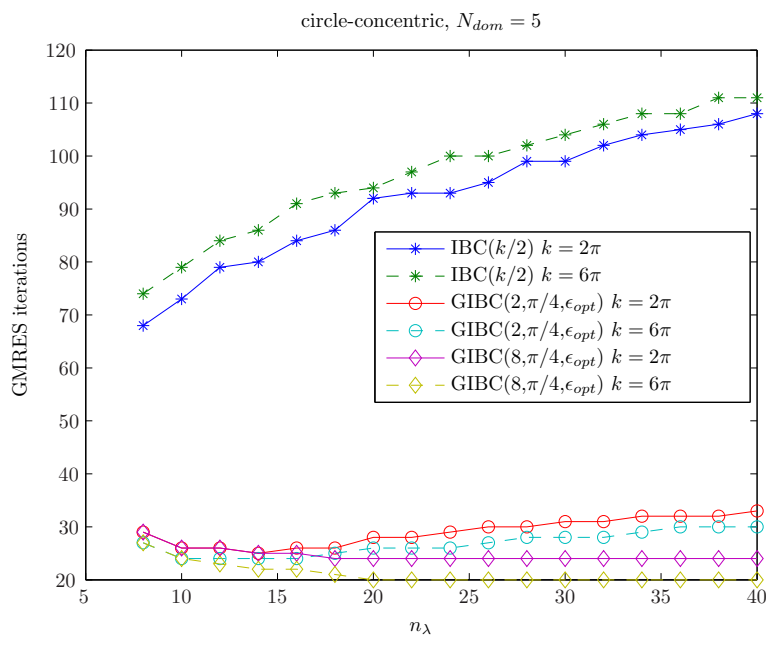

(b) Number of iterations vs. mesh density

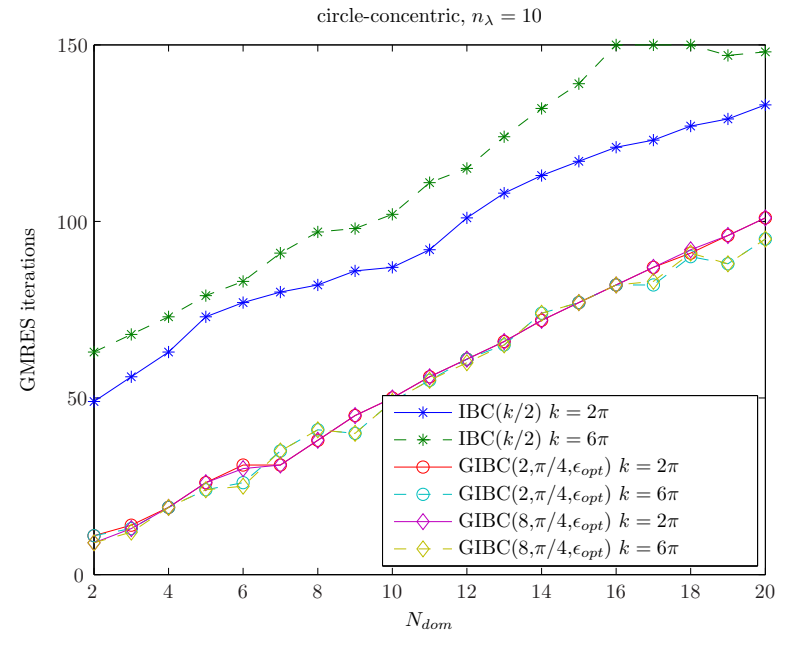

(c) Number of iterations vs. number of subdomains

Figure 7: Convergence for the "circle-concentric" decomposition. 


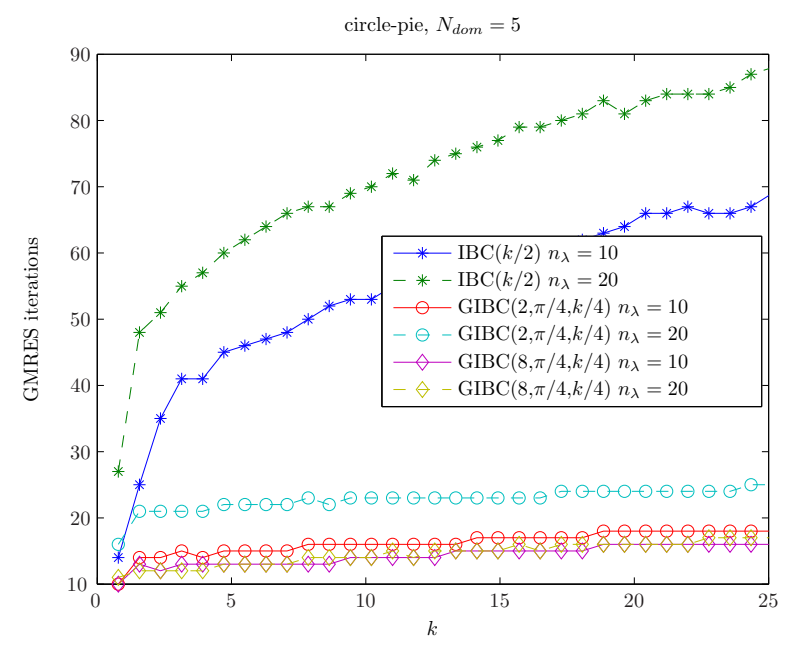

(a) Number of iterations vs. wavenumber

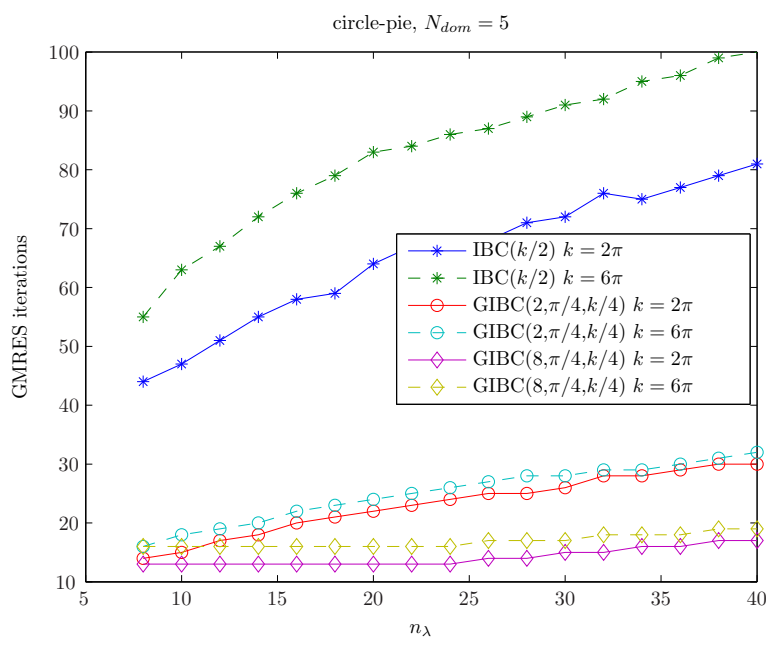

(b) Number of iterations vs. mesh density

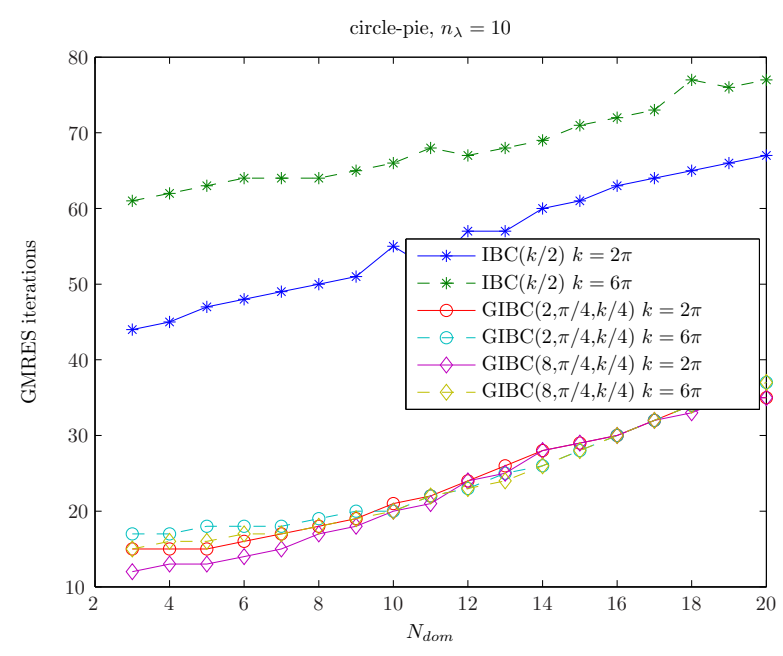

(c) Number of iterations vs. number of subdomains

Figure 8: Convergence for the "circle-pie" decomposition. 


\begin{tabular}{|c|c|c|}
\hline & Number of Unknowns & Factorization Time $(\mathrm{s})$ \\
\hline $\operatorname{IBC}(k / 2)$ & 20812 & 0.37 \\
$\operatorname{GIBC}(1, \pi / 4, k / 4)$ & 21053 & 0.37 \\
$\operatorname{GIBC}(2, \pi / 4, k / 4)$ & 21294 & 0.38 \\
$\operatorname{GIBC}(4, \pi / 4, k / 4)$ & 21776 & 0.39 \\
$\operatorname{GIBC}(8, \pi / 4, k / 4)$ & 22740 & 0.40 \\
$\operatorname{GIBC}(16, \pi / 4, k / 4)$ & 24668 & 0.45 \\
$\operatorname{GIBC}(32, \pi / 4, k / 4)$ & 28524 & 0.53 \\
\hline
\end{tabular}

Table 2: Number of degrees of freedom and computational time for the initial factorization for one subdomain of the "circle-pie" decomposition, with $N_{\mathrm{dom}}=5, k=8 \pi$ and $n_{\lambda}=10$.

penalizing the computational cost of the overall method. Let us also mention that increased values of $n_{\lambda}$ are not only necessary for accuracy purposes but are also imposed to avoid pollution effects in the calculation of the solution at high wavenumbers [31, 33, 32, 8]. Figure $7(\mathrm{~b})$ gives the number of iterations with respect to the density of discretization points per wavelength $n_{\lambda}$, for two wavenumbers $k$. A convergence independent of the mesh size can be achieved provided that $N_{p}$ is sufficiently large, although a very small value $\left(N_{p}=2\right)$ already provides a quasi-optimal result.

In Figure 7(c) we report the number of iterations of the GMRES DDM with respect to the number of subdomains $N_{\text {dom. }}$. We directly see that all the methods scale, for IBC as well as GIBC transmission conditions. Again, for $\operatorname{GIBC}\left(N_{p}, \pi / 4, \varepsilon_{o p t}\right)$, the scaling does not depend on $k$ (and $N_{p}$ ), while this is not the case for $\operatorname{IBC}(k / 2)$.

As a partial conclusion, we can state that using the GMRES algorithm with $\operatorname{GIBC}\left(N_{p}, \pi / 4, \varepsilon_{o p t}\right)$ in the DDM leads to a quasi-optimal convergence: the convergence rate is independent of both the wavenumber $k$ and the density $n_{\lambda}$. Furthermore, the method scales with the number $N_{\text {dom }}$ of subdomains. These conclusions are confirmed by analyzing the behavior of our algorithms for the "circle-pie" decomposition (see Figure 6(b)). Since we have straight interfaces we cannot consider our optimized value of $\varepsilon_{\text {opt }}$ anymore. However, numerical simulations show that $\varepsilon_{\text {opt }}=k / 4$ is a suitable choice. In figures 8(a)$8(\mathrm{c})$, we represent the same three sets of curves as for the "circle-concentric" decomposition. We get a convergence independent of the wave number $k$ and density of discretization $n_{\lambda}$ (by taking $N_{p}$ large enough but relatively small). Again, the algorithm scales with respect to $N_{\mathrm{dom}}$. More generally, the convergence results are even better for the "circle-pie" than for the "circle-concentric" decomposition.

Let us remark here that in the context of the Krylov iterative solution of integral equations for acoustic scattering, quasi optimal convergence independent of the wavenumber and density of discretization points per wavelength was obtained in $[3,4,5]$ by considering Generalized Combined Field Integral Equations including the square-root operator.

\subsubsection{Computational Cost}

The overall computation time of the DDM depends on the number of iterations and the cost of solving (52) for each subdomain. As explained in Section 6, we use a direct sparse linear solver, and the main cost in solving (52) is thus the initial computation of the LU factorization of the augmented matrices of size $N=\left(n_{v}+\left(1+N_{p}\right) n_{t}^{\partial}\right) \times\left(n_{v}+\left(1+N_{p}\right) n_{t}^{\partial}\right)$. We report in Table 2 the number of unknowns $N$ and the factorization time for one subdomain in the case of the "circle-pie" decomposition, with $N_{\text {dom }}=5$, $k=8 \pi$ and $n_{\lambda}=10$. The factorization is performed using MUMPS [1] on a $2.66 \mathrm{GHz}$ MacBook Pro laptop computer. For the typical values of $N_{p}$ required to obtain quasi-optimal convergence $\left(N_{p}=2\right.$ or $N_{p}=8$ ), we see that the computational overhead is small (less than 10\%). 


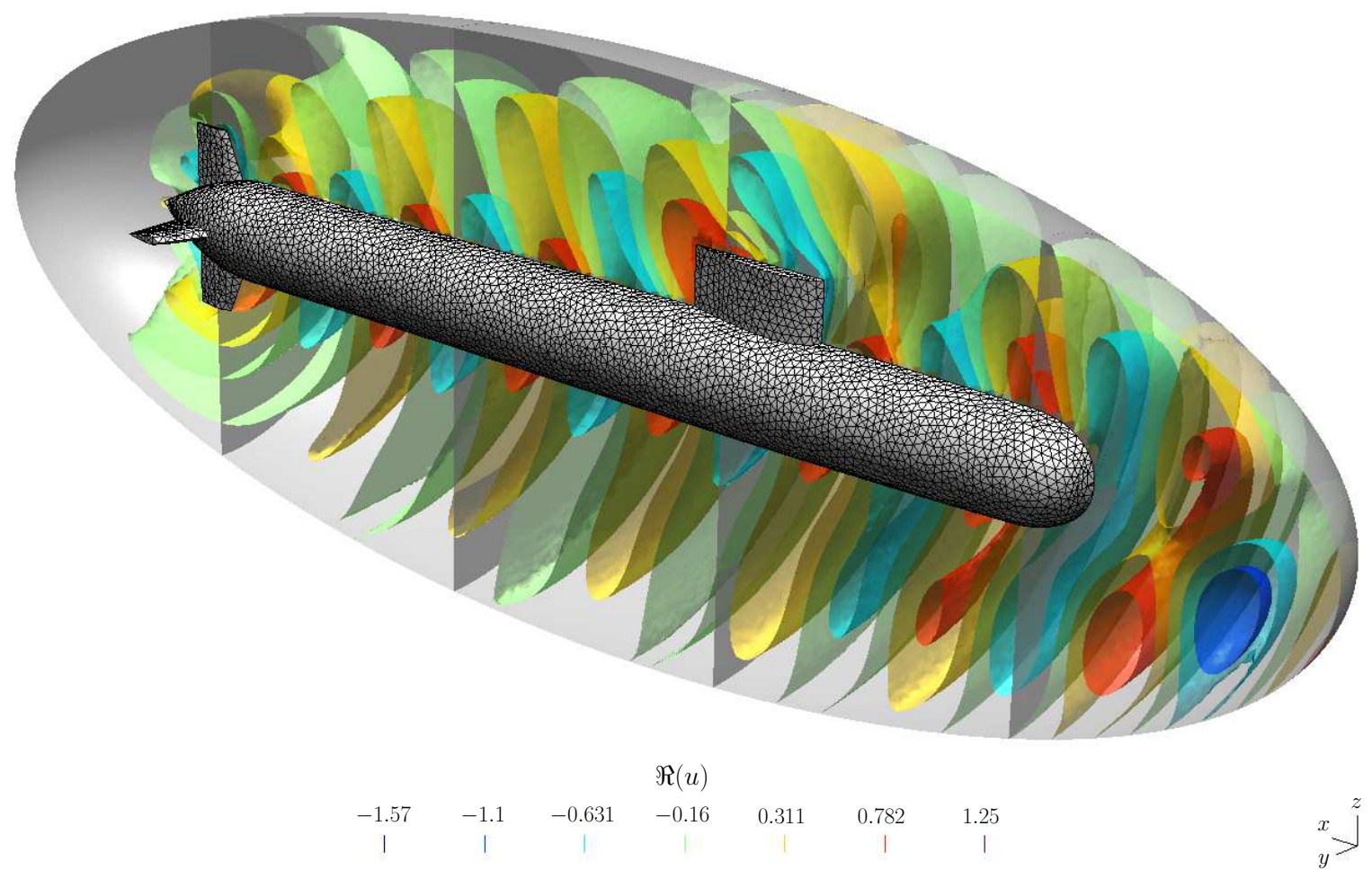

Figure 9: Submarine problem with 5 subdomains: iso-surfaces of the real part of the scattered field for $k=14 \pi$.

\subsection{Three-Dimensional Example}

We now test our algorithm on a more realistic three-dimensional example: the scattering by a submarine. The geometry is represented in Figure 9. For comparison purposes, we choose a unit length submarine. The incident wave is a plane wave with $\boldsymbol{\alpha}=(\cos \pi / 8,0, \sin \pi / 8)^{T}$. We consider two high wave numbers, $k=14 \pi$ and $k=28 \pi$, a density of discretization points per wavelength $n_{\lambda}=10$ and we fix $N_{\text {dom }}=5$ subdomains. The scattered field computed with the GMRES DDM and Padé-type transmission boundary condition is represented on Figure 9. Figure 10 reports the residual decay for different transmission conditions and parameter values. Since we have a plane interface here, we cannot consider our optimized value of $\varepsilon_{\text {opt }}$. As for the "circle-pie" decomposition analyzed in Section 7.1, numerical simulations show that $\varepsilon_{\text {opt }}=k / 4$ is a suitable choice. We see that $\operatorname{GIBC}\left(N_{p}, \pi / 4, \varepsilon_{\text {opt }}\right)$ leads to a very good convergence rate of the GMRES, and is clearly better than EMDA (with $\operatorname{IBC}(k / 2)$ ). For this three-dimensional case, an optimal value of $N_{p}$ to get the quasi-optimality is $N_{p}=8$.

\section{Conclusion}

We have proposed a quasi-optimal non overlapping domain decomposition algorithm for the Helmholtz equation. It is based on a suitable approximation of the DtN operator. A convergence analysis for a model problem has been developed, showing the main features of our algorithm. Several numerical tests in both two- and three dimensions validates the numerical quasi-optimality of the proposed algorithm. Extension to Maxwell's equations is currently being developed. 


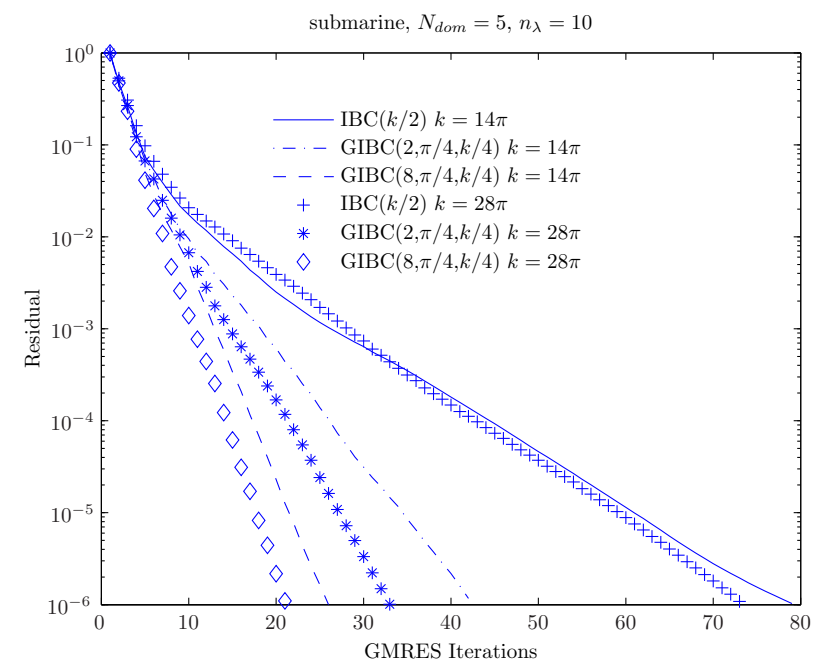

Figure 10: Convergence of the GMRES DDM solvers for the submarine problem.

\section{Acknowledgments}

Y. Boubendir gratefully acknowledges support from NSF through grant No. DMS-1016405. X. Antoine gratefully acknowledges support from the Agence Nationale pour la Recherche (Ref : ANR-09BLAN-0057-01) (http://microwave.math.cnrs.fr/) and the Fondation de Recherche pour l'Aéronautique et l'Espace (IPPON Project). C. Geuzaine gratefully acknowledges support from the Belgian Science Policy (IAP P6/21), Belgian French Community (ARC 09/14-02) and Walloon Region (WIST3 No 1017086 "ONELAB").

\section{References}

[1] P. R. Amestoy, I. S. Duff, J. Koster and J.-Y. L'Excellent, A Fully Asynchronous Multifrontal Solver Using Distributed Dynamic Scheduling, SIAM Journal on Matrix Analysis and Applications, 23 (1), (2001), pp. 15-41.

[2] X. Antoine, H. Barucq and A. Bendali, Bayliss-Turkel-like Radiation Condition on Surfaces of Arbitrary Shape, Journal of Mathematical Analysis and Applications 229 (1999), pp.184-211.

[3] X. Antoine and M. Darbas, Alternative Integral Equations for the Iterative Solution of Acoustic Scattering Problems, Quarterly Journal of Mechanics and Applied Mathematics, 58 (1) (2005), pp. 107-128.

[4] X. Antoine and M. Darbas, Generalized Combined Field Integral Equations for the Iterative Solution of the Three-Dimensional Helmholtz Equation, Mathematical Modelling and Numerical Analysis, 41 (1) (2007), pp. 147-167.

[5] X. Antoine and M. Darbas, Integral Equations and Iterative Schemes for Acoustic Scattering Problems, Chapter book to appear.

[6] X. Antoine, M. Darbas, and Y.Y. Lu, An Improved Surface Radiation Condition for High-Frequency Acoustics Scattering Problems, Computer Methods in Applied Mechanics and Engineering 195 (3336) (2006), pp. 4060-4074. 
[7] X. Antoine, P. Dreyfuss and K. Ramdani, A Construction of Beam Propagation Methods for Optical Waveguides, Communications in Computational Physics 6 (3) (2009), pp. 565-576.

[8] X. Antoine and C. Geuzaine, Phase Reduction Models for Improving the Accuracy of the Finite Element Solution of Time-Harmonic Scattering Problems I: General Approach and Low-Order Models, Journal of Computational Physics 228 (8), (2009), pp.3114-3136.

[9] A. Bayliss, M. Gunzburger and E. Turkel, Boundary Conditions for the Numerical Solutions for Elliptic Equations in Exterior Regions, SIAM Journal of Applied Mathematics 42 (1982), pp.430451.

[10] A. Bayliss and E. Turkel, Radiation Boundary-Conditions for Wave-Like Equations, Communications on Pure and Applied Mathematics, 33 (6) (1980), pp.707-725.

[11] A. Bendali and Y. Boubendir, Non-Overlapping Domain Decomposition Method for a Nodal Finite Element Method, Numerisch Mathematik 103 (4) (2006), pp.515-537.

[12] A. Bendali, Y. Boubendir and M'B. Fares, A FETI-like Domain Decomposition Method for Coupling FEM and BEM in Large-Size Problems of Acoustic Scattering, Computer \& Structures 85 (2007), pp.526-535.

[13] A. Bermudez, L. Hervella-Nieto, A. Prieto and R. Rodriguez, An Exact Bounded Perfectly Matched Layer for Time-Harmonic Scattering Problems, SIAM Journal of Scientific Computing, 30 (1), (2007), pp.312-338.

[14] A. De La Bourdonnaye, C. Farhat, A. Macedo, F. Magoulès and F.X. Roux, A Non Overlapping Domain Decomposition Method for the Exterior Helmholtz Problem, Contemporary Mathematics 218(2) (1998), pp.42-66.

[15] Y. Boubendir. An Analysis of the BEM-FEM Non-Overlapping Domain Decomposition Method for a Scattering Problem, Journal of Computational and Applied Mathematics 204 (2) (2007), pp.282-291.

[16] Y. Boubendir, A. Bendali and M. B. Fares, Coupling of a Non-Overlapping Domain Decomposition Method for a Nodal Finite Element Method with a Boundary Element Method, International Journal for Numerical Methods in Engineering 73 (2008), pp.1624-1650.

[17] F. Collino, S. Ghanemi and P. Joly, Domain Decomposition Method for Harmonic Wave Propagation: a General Presentation, Computer Methods in Applied Mechanics and Engineering 184, (2000), pp.171-211.

[18] F. Collino and P. Monk, The Perfectly Matched Layer in Curvilinear Coordinates, SIAM Journal of Scientific Computing 19 (6) (1998), pp.2061-2090.

[19] B. Després, Méthodes de Décomposition de Domaine pour les Problèmes de Propagation d'Ondes en Régime Harmonique. Le Théorème de Borg pour l'Equation de Hill Vectorielle, PhD Thesis, Paris VI University, France, 1991.

[20] B. Després, Domain Decomposition Method and the Helmholtz Problem, 1991; Mathematical and numerical aspects of wave propagation phenomena, 44-52, Strasbourg, G. Cohen, L. Halpern and P. Joly. SIAM, Philadelphia, PA.

[21] B. Després, Domain Decomposition Method and the Helmholtz Problem (part II), 1993; Mathematical and numerical aspects of wave propagation phenomena, pages 197-206, SIAM, Philadelphia, PA. 
[22] P. Dular, C. Geuzaine, F. Henrotte and W. Legros. A General Environment for the Treatment of Discrete Problems and its Application to the Finite Element Method, IEEE Transactions on Magnetics, 34 (5), (1998), pp. 3395-3398.

[23] B. Engquist and A. Majda, Absorbing Boundary Conditions for the Numerical Simulation of Waves, Mathematics of Computation 23 (1977), pp.629-651.

[24] C. Farhat, A. Macedo and M. Lesoinne, A Two-Level Domain Decomposition Method for the Iterative Solution of High Frequency Exterior Helmholtz Problems, Numerisch Mathematik 85(2) (2000), pp.282-303.

[25] C. Farhat, A. Macedo, M. Lesoinne, F.X. Roux, F. Magoulès and A. De La Bourdonnaye, TwoLevel Domain Decomposition Methods with Lagrange Multipliers for the Fast Iterative Solution of Acoustic Scattering Problems, Computer Methods in Applied Mechanics and Engineering 184 (2000), pp.213-239.

[26] C. Farhat, R. Tezaur and J. Toivanen, A Domain Decomposition Method for Discontinuous Galerkin Discretizations of Helmholtz Problems with Plane Waves and Lagrange Multipliers, International Journal for Numerical Methods in Engineering 78 (2009), pp.1513-1531.

[27] M.J. Gander, F. Magoulès and F. Nataf, Optimized Schwarz Methods without Overlap for the Helmholtz Equation, SIAM Journal of Scientific Computing, 24 (1) (2002), pp. 38-60.

[28] C. Geuzaine and J.-F. Remacle, Gmsh: a Three-Dimensional Finite Element Mesh Generator with Built-in Pre- and Post-Processing Facilities, International Journal for Numerical Methods in Engineering 79 (11), (2009), pp. 1309-1331.

[29] M-N. Guddati and K.-W Lim, Continued Fraction Absorbing Boundary Conditions for Convex Polygonal Domains, International Journal for Numerical Methods in Engineering 66 (2006), pp. 949977

[30] P.L. Ho and Y.Y. Lu, Improving Beam Propagation Method for TM polarization, Optics and Quantum Electronics, 35 (4), (2003), pp. 507-519.

[31] C. Ianculescu and L.L. Thompson, Parallel Iterative Solution for the Helmholtz Equation with Exact Non-Reflecting Boundary Conditions, Computer Methods in Applied Mechanics and Engineering 195 (29-32), (2006), pp.3709-3741.

[32] F. Ihlenburg and I. Babuska, Dispersion Analysis and Error Estimation of Galerkin Finite Element Methods for the Helmholtz Equation, International Journal for Numerical Methods in Engineering, 38 (1995), pp.3745-3774.

[33] F. Ihlenburg, Finite Element Analysis of Acoustic Scattering, Springer-Verlag, 1998.

[34] R. Kerchroud, X. Antoine and A. Soulaimani. Numerical Accuracy of a Padé-Type Non-Reflecting Boundary Condition for the Finite Element Solution of Acoustic Scattering Problems at HighFrequency, International Journal for Numerical Methods in Engineering 64 (10) (2005), pp. 12751302.

[35] R. Kerchroud, A. Soulaimani and X. Antoine, Performance Study of Plane Wave Finite Element Methods with a Padé-Type Artificial Boundary Condition in Acoustic Scattering, Advances in Engineering Software 40 (2009), pp.738-750.

[36] P.-L. Lions. On the Schwarz Alternating Method III: A Variant for Non Overlapping Subdomains, Third International Symposium on Domain Decomposition Methods for Partial Differential Equations, held in Houston, Texas, March 20-22,(1989), 1990; T.F. Chan, R. Glowinski, J. Périaux and O. Widlund, SIAM, Philadelphia, PA. 
[37] Y.Y Lu, Some Techniques for Computing Wave Propagation in Optical Waveguides, Communications in Computational Physics, 1 (6), (2006), pp. 1056-1075.

[38] F. A. Milinazzo, C. A. Zala and G. H. Brooke, Rational Square-Root Approximations for Parabolic Equation Algorithms, Journal of the Acoustical Society of America 101(2) (1997), pp.760-766.

[39] A. Quarteroni and A. Valli, Domain Decomposition Methods for Partial Differential Equations, Oxford University Press, 1999.

[40] F. X. Roux, F. Magoules, L. Series and Y. Boubendir, Approximation of Optimal Interface Boundary Conditions for Two-Lagrange Multiplier FETI Method, Lecture Notes in Computational Science and Engineering, Volume 40, 2005, Pages 283-290.

[41] F.-X. Roux, F. Magoulès, S. Salmon, and L. Series, Optimization of Interface Operator Based on Algebraic Approach, In Domain Decomposition Methods in Sci. Engrg., pages 297304, 2002.

[42] Y. Saad. Iterative Methods for Sparse Linear Systems, PWS, Boston, 1996.

[43] T. B. A. Senior and J. L. Volakis, Approximate Boundary Conditions in Electromagnetics, IEE Press, New York and London, 1995.

[44] I. Singer and E. Turkel, A Perfectly Matched Layer for the Helmholtz Equation in a Semi-Infinite Strip, Journal of Computational Physics 201 (2), (2004), pp.439-465.

[45] B. Stupfel, A Hybrid Finite Element and Integral Equation Domain Decomposition Method for the Solution of the 3-D Scattering Problem, Journal of Computational Physics 172 (2) (2001), pp.451-471.

[46] M. E. Taylor, Pseudodifferential Operators, Princeton University Press, Princeton, New Jersey (1981).

[47] A. Toselli and O. Windlund, Domain Decomposition Methods Algorithms and Theory, Vol. 34, Springer Series in Computational Mathematics, Springer, 2004.

[48] E. Turkel and A. Yefet, Absorbing PML Boundary Layers for Wave-Like Equations, Applied Numerical Mathematics, 27 (4), (1998), pp.533-557. 